MANNHEIM RESEARCH INSTITUTE FOR THE ECONOMICS OF AGING

\title{
A Parsimonious Model of SubJective Life EXPECTANCY
}

Alexander Ludwig and Alexander Zimper

154-2007

๑ mea-Mannheim Research Institute for the Economics of Aging

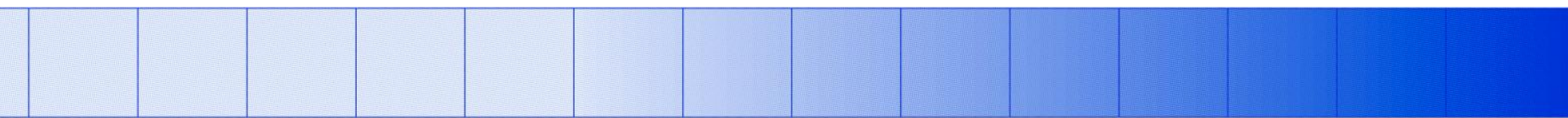

L13, 17_D-68131 Mannheim_Phone +49 621 181-2773/1862_Fax +49 621 181-1863_www.mea.uni-mannheim.de 


\title{
A Parsimonious Model of Subjective Life Expectancy*
}

\author{
Alexander Ludwig ${ }^{\dagger} \quad$ Alexander Zimper ${ }^{\ddagger}$
}

November 6, 2007

\begin{abstract}
This paper develops a theoretical model for the formation of subjective beliefs on individual survival expectations. Data from the Health and Retirement Study (HRS) indicate that, on average, young respondents underestimate their true survival probability whereas old respondents overestimate their survival probability. Such subjective beliefs violate the rational expectations paradigm and are also not in line with the predictions of the rational Bayesian learning paradigm. We therefore introduce a model of Bayesian learning which combines rational learning with the possibility that the interpretation of new information is prone to psychological attitudes. We estimate the parameters of our theoretical model by pooling the HRS data. Despite a parsimonious parametrization we find that our model results in a remarkable fit to the average subjective beliefs expressed in the data.
\end{abstract}

JEL classification: C44, D83, D91, I10

Keywords: subjective survival expectations; ambiguity; Bayesian learning

*We thank Axel Börsch-Supan, Andrew Caplin, Mike Hurd, Jürgen Maurer, Susan Rohwedder, Aylit Romm, Martin Salm, Carsten Trenkler, David Weir and Joachim Winter as well as several seminar participants at the Workshop on Subjective Probabilities and Expectations, Jackson Hole, Wyoming, 2007 and at Universität Mannheim for helpful comments and Frank Schilbach for his excellent research assistance. Financial support by the Social Security Administration (SSA) under the 2007 Steven H. Sandell Grant Program, the German National Research Foundation (DFG) through SFB 504, the State of Baden-Württemberg and the German Insurers Association (GDV) is gratefully acknowledged.

${ }^{\dagger}$ Mannheim Research Institute for the Economics of Aging (MEA); Universität Mannheim; L13, 17; 68131 Mannheim; Germany; Email: ludwig@mea.uni-mannheim.de.

${ }^{\ddagger}$ Department of Economics; University of Leicester; University Road; LE1 7RH; United Kingdom; Email: zimper@bigfoot.com. 


\section{Introduction}

Dynamic economic models are based on the forward looking behavior of economic agents. In the context of life-cycle models, an individual's consumption and savings decision depends on her subjective beliefs about future interest rates, wage rates and the likelihood of dying. According to these models, individuals have beliefs about such variables and use these beliefs to make decisions today. Until recently common practice in such studies was to assume rational expectations implying that the individuals' beliefs are given as objective probability distributions. The use of objective distributions is by now put into question by numerous researchers who suggest to directly measure subjective expectations and to evaluate the consequences of deviations of subjective expectations from their objective counterparts. Manski (2004) provides an overview on this literature.

In this paper we develop a model of survival belief formation that differs from the existing literature on subjective expectations in two respects. First, we allow for the possibility that people report subjective beliefs that express ambiguity attitudes. We will formally describe such ambiguous beliefs as non-additive probability measures, i.e., capacities, which arise in Choquet Expected Utility (CEU) theory (Schmeidler 1989; Gilboa and Schmeidler 1993) in order to accommodate Ellsberg paradoxes (Ellsberg 1961). Second, as a generalization of the standard assumption of rational Bayesian learning, we consider a model of psychologically biased Bayesian learning. For the representative agent of our model an initial bias between her subjective beliefs and objective probabilities does not necessarily vanish in the long run. Several studies in the psychological literature show that real-life agents systematically violate the assumption of rational Bayesian learning in that their learning behavior is prone to effects such as "myside bias" or "irrational belief persistence" cf. Baron (2007, Ch. 9) and the references cited there. The stylized facts in our data on subjective survival beliefs may reflect such attitudes and our formal approach attempts to accommodate these.

As our point of departure we compare subjective survival beliefs, based on the data of the last three waves of the Health and Retirement Study (HRS), with objective survival rates. In our data on subjective beliefs we have information about individuals' expectations to live from the age at interview $j$ up to some target age $m$. Age at interview $j$ and target age $m$ are assigned according to the pattern in table 1 . Our objective survival rates are based on cohort life tables for the U.S. population. ${ }^{1}$ We also address the concern of selectivity of our HRS sample and provide empirical evidence that population wide mortality rates can indeed be used as the relevant objective data in our context.

Figure 1 summarizes the information in our data by displaying the average subjective beliefs on survival of HRS respondents against the age at interview and the

\footnotetext{
${ }^{1}$ To construct these cohort life tables we predict population wide survival probabilities taken from the Social Security Administration (SSA) and the Human Mortality Database (HMD) beyond the data horizon of 2004, the year of the last wave of the HRS. We base the extrapolation on estimates stemming from a Lee-Carter procedure (Lee and Carter 1992).
} 
Table 1: Interview and Target Age

\begin{tabular}{c|c}
\hline \hline Age at Interview $j$ & Target Age $m$ \\
\hline$\leq 69$ & 80 \\
$70-74$ & 85 \\
$75-79$ & 90 \\
$80-84$ & 95 \\
$85-89$ & 100 \\
\hline \hline
\end{tabular}

Source: RAND HRS Data Documentation, Version F (October 2006).

respective objective data for men in panel (a) and women in panel (b). The different line segments are due to changes in target ages, cf. table 1. Two stylized facts emerge for either gender from the data. First, the subjective beliefs on survival are downward biased at younger ages. Second, the subjective beliefs on survival are upward biased at older ages whereby the upward bias increases with age. These stylized facts clearly indicate a systematic violation of the rational expectations paradigm of economic theory by which there should be no difference between subjective beliefs and objective survival rates. ${ }^{2}$

In addition to the violation of the rational expectations paradigm, the age-dependent bias in the HRS data about life expectancy also suggests a violation of the rational Bayesian learning paradigm. Models of subjective belief formation based on rational Bayesian learning generate posterior beliefs that are closer to the true, i.e., objective, distribution the more experienced the agent becomes. If an agent gains more experience by getting older, rational Bayesian learning requires the agent to learn with increasing age the true probabilities (cf. Viscusi $(1990,1991)$ and subsection 2.2 of this paper for a more detailed discussion). Under the assumption of rational Bayesian learning any gap between subjective beliefs and objective survival probabilities should therefore decrease with increasing age, implying that the average beliefs of people are closer to the true probabilities when they get older. For younger respondents $(\leq 69)$ the data in figure 1 is compatible with the convergence behavior as predicted by rational Bayesian learning. ${ }^{3}$ However, upon inspection of the age-belief pattern of elderly respondents of age 75 and older in figure 1, the picture changes. In figure 2 we zoom in from figure 1 the average beliefs of male respondents between interview ages 80 to 89 to survive

\footnotetext{
${ }^{2}$ Our findings thus confirm similar results belonging to a line of research initiated by Hammermesh (1985). In two different data samples from surveys, Hammermesh (1985) found that people do incorporate improvements of life-expectancy into their beliefs about personal longevity and that the subjective survival curves are somewhat flatter than the objective data. Similar differences between subjective beliefs and the objective data have been reported for the HRS by Hurd and Kathleen (1995) and Gan, Hurd, and McFadden (2003) and others and, more recently, for the Survey of Health, Ageing and Retirement in Europe (SHARE) data (Hurd, Rohwedder, and Winter 2005).

${ }^{3}$ For women we do not observe such a clear convergent pattern even for this age group.
} 
Figure 1: Subjective and objective survival probabilities

(a) Men

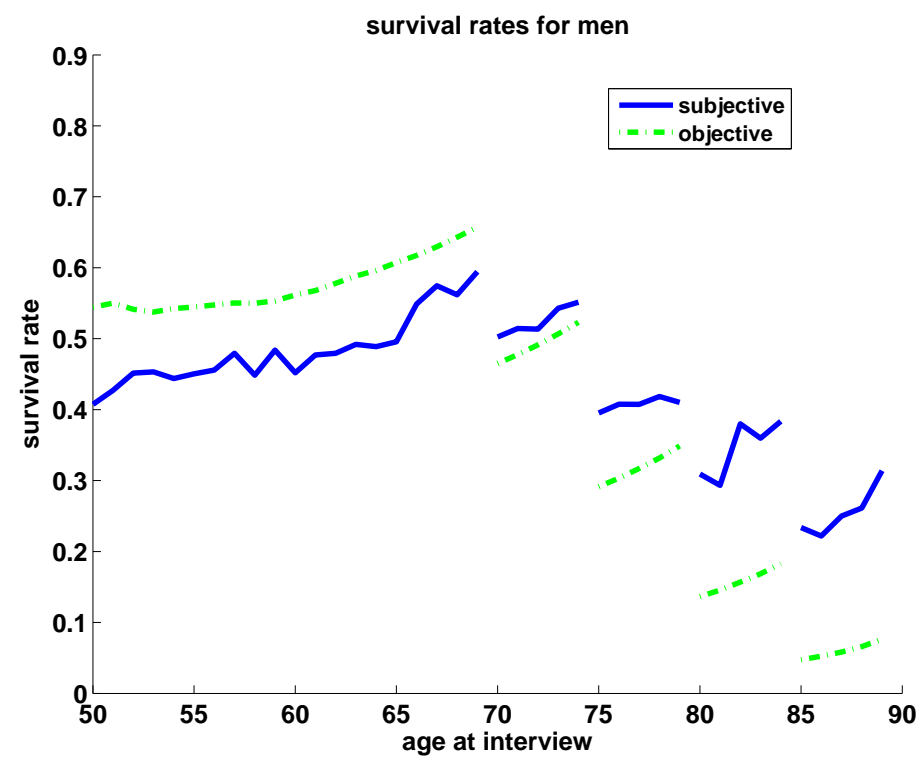

(b) Women

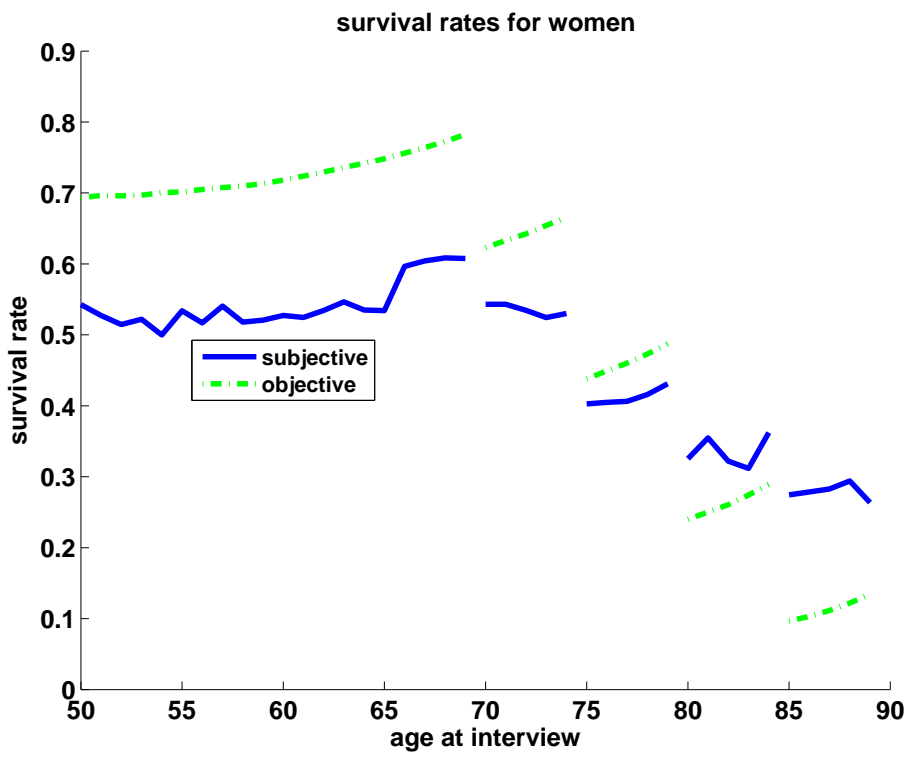

Source: Own calculations based on HRS, HMD and SSA data. 
until 95, respectively until 100, against their objective counterparts in panel (a). To illustrate learning behavior in this age group we estimated simple linear trends for both the subjective and the objective data and display the differences in these trends in panel (b) of the same figure. This graphs indicates divergence with increasing age. This divergent pattern is stronger for the higher interview/target age group. Thus, contrary to the predictions of the rational Bayesian learning model the average bias between subjective beliefs and objective probabilities increases rather than decreases with more experience whereby this effect appears to be stronger for higher target ages.

The patterns shown in figure 2 illustrate a violation of the rational Bayesian learning paradigm within target age groups. Furthermore, notice that, in order to explain the data across target age groups, the rational Bayesian learning hypothesis would require highly implausible prior beliefs. For example, the overestimation of the subjective belief of an 80 year old agent to live until 95 by 17.28 percentage points for men (8.54 percentage points for women), cf. figure 1, can only be explained with rational Baysian learning if the same agent expressed a prior belief with a much higher degree of overestimation about her survival at the age of 50. However, at the age of 50, we actually observe an average underestimation of the survival belief by -13.70 percentage points for men ( -15.07 percentage points for women).

Our model of Bayesian learning with psychological bias captures the stylized facts of figure 1 in a very parsimonious way whereby it also offers a plausible explanation why young people are too pessimistic whereas elderly people are too optimistic about their survival expectations. While rational Bayesian learning may be appropriate in situations in which individuals are emotionally detached from any new information arriving (think, e.g., about tossing a coin in order to learning the odds whether it ends up heads or tails), this may not be the case if new information has a strong personal impact on the individual. In such situations the individual's learning process may be prone to emotions such as hope or despair. This holds in particular true when an individual learns new information about its life expectancy thereby facing the prospect of its own death. ${ }^{4}$ We feel that the most plausible explanation for the overly optimistic life expectations of elderly people is an optimistic "myside bias" in their interpretation of any new information which assists them to better ignore the increasingly relevant prospect of death. In contrast, younger people are less biased because the prospect of their death is less relevant yet and they may even underestimate increases in life expectancy due to medical progress.

Our theoretical framework provides a parsimonious specification of the representative agent's age-belief pattern with four parameters, reflecting, first, an initial bias in subjective survival probabilities, second, the strength of the rational Bayesian learning process, third, ambiguity aversion, and, fourth, the degree of optimism, respectively pessimism, by which the agent resolves her ambiguity. We then estimate the parameters

\footnotetext{
${ }^{4}$ Along this line, Kastenbaum (2000) summarizes the insights of psychological research on the reflection about personal death as follows: "There are divergent theories and somewhat discordant findings, but general agreement that most of us prefer to minimize even our cognitive encounters with death."
} 
Figure 2: Survival probabilities at age 80 and older for men

(a) Objective and subjective probabilities

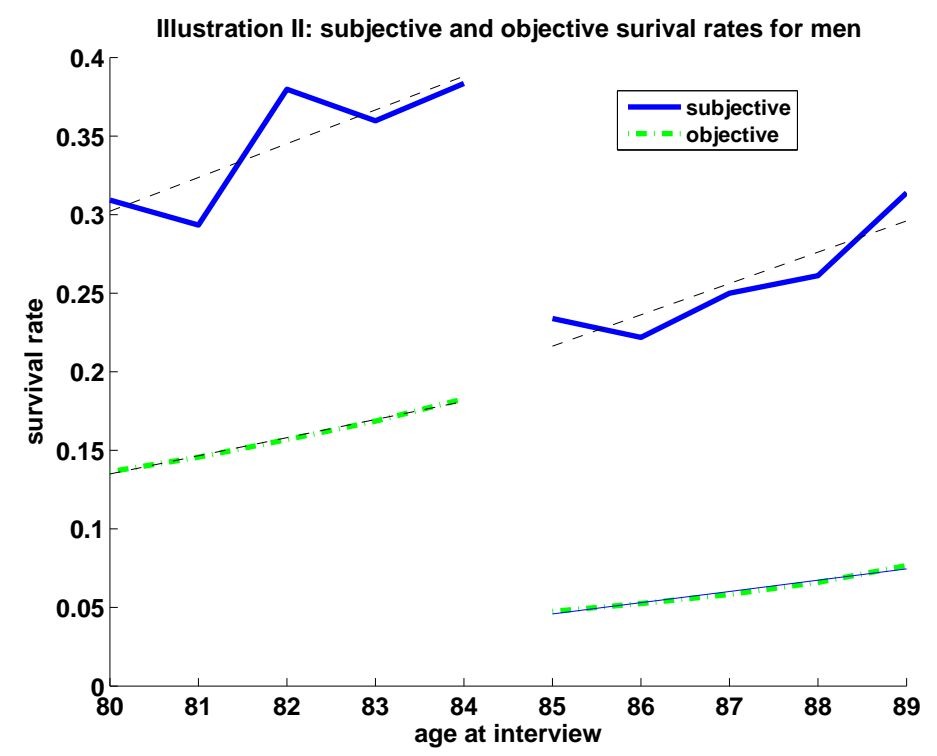

(b) Difference in trends

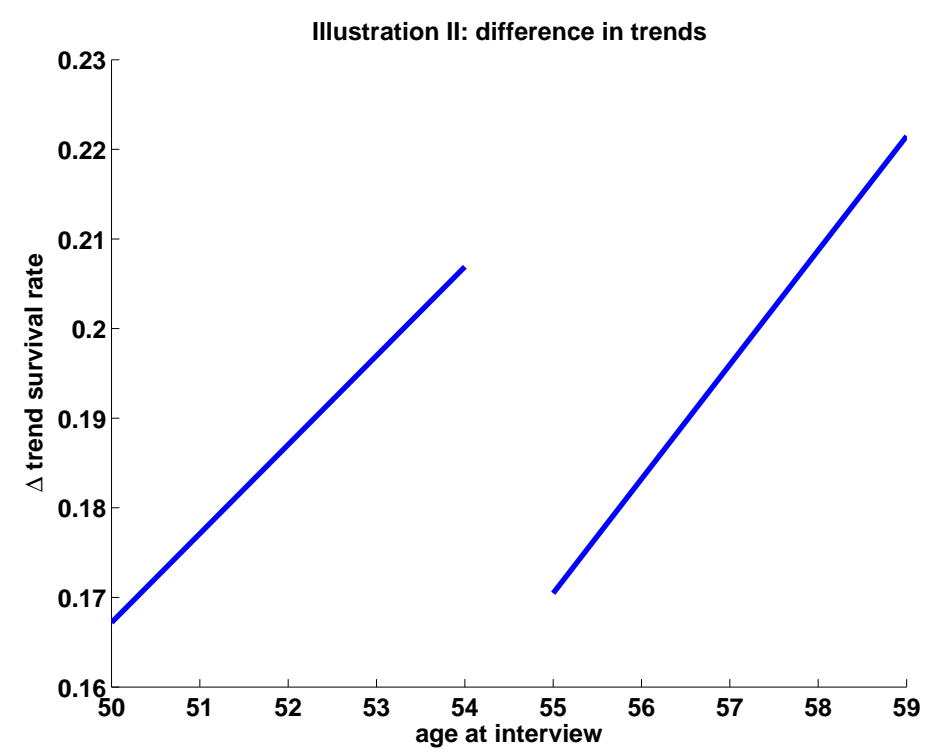

Notes: The dashed lines in panel (a) are predicted values from a simple linear trend estimation. Panel (b) displays the difference in these linear trends.

Source: Own calculations based on HRS, HMD and SSA data. 
of our theoretical model by pooling the HRS data. Despite the low parametrization, our model results in a decent fit to the average data on subjective beliefs. We also find that the model's performance is somewhat better for female than for male respondents.

Our approach is related to a literature initiated by Viscusi (1985) who analyzes changes in risk perceptions by a simple model of rational Bayesian learning. In the context of the HRS data on subjective survival probabilities this approach has been used by Smith, Taylor, and Sloan (2001) and Smith, Taylor, Sloan, Johnson, and Desvouges (2001) who test how new information about health shocks between two interview waves affects updating of individuals in the HRS. ${ }^{5}$ In contrast to this literature our approach is more general in that it allows for the possibility that individuals are not rational Bayesian learners. Moreover, while we do not investigate how certain idiosyncratic shocks, e.g., general versus smoking related health shocks or parental death, affect updating of individuals and how updating differs across subgroups of individuals, the strength of our parsimonious setup is that it can be directly mapped into calibrated micro- or macroeconomic life-cycle models with representative agents.

The remainder of our analysis is structured as follows. Section 2 presents our decision theoretic framework resulting in a parsimonious model of subjective life expectancy. In section 3 we describe the data we use for estimation of the parameters of our model. We then present the main results of our empirical analysis in section 4 . Finally, section 5 concludes. A separate appendix contains proofs and some additional results on sensitivity analysis.

\section{A Parsimonious Model of Subjective Life Expectancy}

\subsection{Ambiguous Beliefs}

We assume that individuals exhibit ambiguity attitudes in the sense of Schmeidler (1989) and who may thus, for example, commit paradoxes of the Ellsberg type (Ellsberg 1961). Following Schmeidler (1989), such individuals could be described as Choquet Expected Utility (CEU) decision makers, that is, they maximize expected utility with respect to non-additive beliefs. ${ }^{6}$ Our own approach focuses on non-additive beliefs that are defined as neo-additive capacities in the sense of Chateauneuf, Eichberger, and Grant (2007).

\footnotetext{
${ }^{5}$ Under the assumption that all individuals are rational Bayesian learners, Smith, Taylor, Sloan, Johnson, and Desvouges (2001) show that a representative smoker updates her beliefs in a different way than a representative non-smokers when she learns about "general" compared to "smoking-related" health shocks.

${ }^{6}$ Properties of non-additive beliefs are used in the literature for formal definitions of, e.g., ambiguity and uncertainty attitudes (Schmeidler 1989; Epstein 1999; Ghirardato and Marinacci 2002), pessimism and optimism (Eichberger and Kelsey 1999; Wakker 2001; Chateauneuf, Eichberger, and Grant 2007), as well as sensitivity to changes in likelihood (Wakker 2004).
} 
Definition 1. For a given measurable space $(S, \Sigma)$ the neo-additive capacity, $\nu$, is defined, for some $\delta, \lambda \in[0,1]$ by

$$
\nu(E)=\delta \cdot\left(\lambda \cdot \omega^{o}(E)+(1-\lambda) \cdot \omega^{p}(E)\right)+(1-\delta) \cdot \tilde{\pi}(E)
$$

for all $E \in \Sigma$ such that $\tilde{\pi}$ is some additive probability measure and we have for the non-additive capacities $\omega^{o}$

$$
\begin{aligned}
& \omega^{o}(E)=1 \text { if } E \neq \emptyset \\
& \omega^{o}(E)=0 \text { if } E=\emptyset
\end{aligned}
$$

and $\omega^{p}$ respectively

$$
\begin{aligned}
& \omega^{p}(E)=0 \text { if } E \neq S \\
& \omega^{p}(E)=1 \text { if } E=S .
\end{aligned}
$$

Recall that a Savage-act $f$ is a mapping from the state space $S$ into the set of consequences $X$. For a finite state space the Choquet expected utility of Savage act $f$ with respect to a neo-additive capacity $\nu$ is given as

$$
\begin{aligned}
C E U(f, \nu)= & \delta \cdot\left(\lambda \cdot \max _{s \in S} u(f(s))+(1-\lambda) \cdot \min _{s \in S} u(f(s))\right) \\
& +(1-\delta) \cdot \sum_{s \in S} \tilde{\pi}(s) \cdot u(f(s))
\end{aligned}
$$

where $u: X \rightarrow \mathbb{R}$ is a von Neumann-Morgenstern utility function. Neo-additive capacities can be interpreted as non-additive beliefs that represent deviations from additive beliefs such that a parameter $\delta$ (degree of ambiguity) measures the lack of confidence the decision maker has in some subjective additive probability distribution $\tilde{\pi}$. Obviously, if there is no ambiguity, i.e., $\delta=0$, equation (2) reduces to the standard subjective expected utility representation of Savage (1954). In case there is some ambiguity, however, the second parameter $\lambda$ measures how much weight the decision maker puts on the best possible outcome of alternative $f$ when resolving her ambiguity. Conversely, $(1-\lambda)$ is the weight she puts on the worst possible outcome of $f$. As a consequence, we interprete $\lambda$ as an "optimism under ambiguity" parameter.

In the context of survival expectations, we are interested in the agent's belief to be alive at some target age $m$. Let us misuse notation and also write $m$ for the event that the agent is still alive at age $m$. Under the assumption that there is always the possibility to reach age $m$, the event $m$ cannot be the null event, implying $\omega^{o}(m)=1$. On the other hand, we also stipulate that there is always the possibility to die before reaching age $m$ so that $m$ cannot be the universal event either, implying $\omega^{p}(m)=0$. As a consequence, the agent's belief to survive until age $m$ in (1) simplifies to

$$
\nu(m)=\delta \cdot \lambda+(1-\delta) \cdot \tilde{\pi}(m)
$$


According to our interpretation the additive probability distribution $\tilde{\pi}$ in (3) is the representative agent's estimator of her survival probability. Under the rational expectations paradigm this estimator $\tilde{\pi}$ must, first, coincide with the "true" probability distribution and, second, the agent must not be ambiguous about her subjective belief, i.e., $\delta=0$. However, we do not only assume that the representative agent is ambiguous about her subjective belief, $\delta \neq 0$, but also that $\tilde{\pi}$ may be a biased estimator of the "true" probability distribution, denoted by $\pi$.

\subsection{Bayesian Learning with Psychological Bias}

In this subsection we develop our concept of Bayesian learning with a psychological bias. In our model the representative agent receives new information about her lifeexpectancy when she gets older. Since we consider ambiguous beliefs, the resulting updating process deviates from rational Bayesian learning in that the strength of ambiguity attitudes may increase over time. We proceed in four steps.

Recall, in a first step, Viscusi (1985)'s model of rational Bayesian learning ${ }^{7}$ and consider some prior Beta distribution over the parameter $\pi$ of a Binomial-distribution such that $\pi$ is the "true" probability that a representative agent survives to age $m$. If the subjective prior about $\pi$, denoted $\tilde{\pi}$, is the expected value of this prior Beta distribution, we have $\tilde{\pi}(m)=\frac{\alpha}{\alpha+\beta}$ for given parameters $\alpha$ and $\beta$. Let $I$ denote information that is equivalent to a statistical experiment in which $m$ has occurred $k$-times in $n$ independent trials and let $\tilde{\pi}_{I}(m)$ denote the agent's posterior about $\pi$ given the new information $I .{ }^{8}$ Rational Bayesian updating then results in a posterior Beta distribution about $\pi$ with expected value $\frac{\alpha+k}{\alpha+\beta+n}$ implying for the posterior belief

$$
\tilde{\pi}_{I}(m)=\gamma_{n} \cdot \tilde{\pi}(m)+\left(1-\gamma_{n}\right) \cdot \mu_{n}(m)
$$

such that

$$
\gamma_{n}=\frac{\alpha+\beta}{\alpha+\beta+n}
$$

where $\mu_{n}(m)$ is the sample mean $\frac{k}{n} \cdot 9$

Rather than modelling the unconditional belief of surviving to some target age our focus is on the agent's conditional survival beliefs. Under the assumption of rational Bayesian learning we therefore look, in a second step, at the belief $\tilde{\pi}_{I}(m \mid j)$ that the agent will be alive at age $m$ provided that she is alive at age $j$ and has received

\footnotetext{
${ }^{7}$ This specific model of rational Bayesian learning has become very popular in the empirical literature on risk perception (Viscusi 1991; Smith, Taylor, and Sloan 2001; Smith, Taylor, Sloan, Johnson, and Desvouges 2001).

${ }^{8}$ As a notational convention we use subscript notation when we condition on the information available to the agent. The reason for this notational convention will become clear once we move from unconditional to conditional expectations.

${ }^{9}$ Tonks (1983) introduces a similar model of rational Bayesian learning in which the agent has a normally distributed prior over the mean of some normal distribution.
} 
information $I$. Since $j \leq m$, we obtain by (4) for this conditional belief

$$
\tilde{\pi}_{I}(m \mid j)=\frac{\tilde{\pi}_{I}(m)}{\tilde{\pi}_{I}(j)}=\gamma_{n}^{*} \cdot \tilde{\pi}(m \mid j)+\left(1-\gamma_{n}^{*}\right) \cdot \frac{\mu_{n}(m)}{\mu_{n}(j)}
$$

with

$$
\gamma_{n}^{*}=\frac{\tilde{\pi}(j) \cdot(\alpha+\beta)}{\tilde{\pi}(j) \cdot(\alpha+\beta)+n \cdot \mu_{n}(j)} .
$$

In a next step, we have to take a stand on how an agent updates her ambiguous beliefs. Several different Bayesian update rules are perceivable for the non-additive beliefs of CEU decision-makers (Gilboa and Schmeidler 1993; Pires 2002; Eichberger, Grant, and Kelsey 2006; Siniscalchi 2001; Siniscalchi 2006). In this paper we consider the so-called generalized Bayesian update rule. As shown in appendix A.1 this results in the posterior conditional survival belief

$$
\nu_{I}(m \mid j)=\delta_{I} \cdot \lambda+\left(1-\delta_{I}\right) \cdot \tilde{\pi}_{I}(m \mid j)
$$

where

$$
\delta_{I}=\frac{\delta}{\delta+(1-\delta) \cdot \tilde{\pi}(j \cap I)} .
$$

In a final step, we link the information received by the agent to her age. We suppose that an agent of age $h$ receives information $I_{h}$ which is equivalent to information gained from a statistical experiment with $n(h)$ trials whereby the experience function $n(h)$ satisfies $n(0)=0$ and $n(h) \rightarrow \infty$ if $h \rightarrow \infty$. That is, our approach associates a higher age with greater experience.

Proposition 1. Under the assumption of Bayesian learning with psychological bias, the posterior belief of an agent of age $h$ to survive until age $m$ conditional on being alive at age $j$ is given as

$$
\nu_{I_{h}}(m \mid j)=\delta_{I_{h}} \cdot \lambda+\left(1-\delta_{I_{h}}\right) \cdot \tilde{\pi}_{I_{h}}(m \mid j)
$$

such that

$$
\delta_{I_{h}}=\frac{\delta}{\delta+(1-\delta) \cdot \tilde{\pi}\left(j \cap I_{h}\right)}
$$

and

$$
\tilde{\pi}_{I_{h}}(m \mid j)=\gamma_{n(h)}^{*} \cdot \tilde{\pi}(m \mid j)+\left(1-\gamma_{n(h)}^{*}\right) \cdot \frac{\mu_{n(h)}(m)}{\mu_{n(h)}(j)}
$$

with

$$
\gamma_{n(h)}^{*}=\frac{\tilde{\pi}(j) \cdot(\alpha+\beta)}{\tilde{\pi}(j) \cdot(\alpha+\beta)+n(h) \cdot \mu_{n(h)}(j)} .
$$


Observe that $\mu_{n}(m) \rightarrow \pi(m)$ and $\mu_{n}(j) \rightarrow \pi(j)$ in probability if the number of trials approaches infinity. Since $\gamma_{n}^{*} \rightarrow 0$ if $n(h) \rightarrow \infty$, the posterior $\tilde{\pi}_{I_{h}}(m \mid j)$ converges in probability to the true survival rate $\pi(m \mid j)$ if the agent observes infinitely many trials. Thus, in the case without ambiguity, i.e., for $\delta=0$, our model reduces to the standard model of rational Bayesian learning. Furthermore, under the plausible assumption that the agent does not expect to live forever, that is for $\tilde{\pi}(j) \rightarrow 0$ with increasing age $j$, we observe the following:

Observation 1. Under Bayesian learning with psychological bias the conditional posterior belief $\nu_{I_{h}}(m \mid j)$ converges in probability with increasing age to the initial degree of optimism under ambiguity, $\lambda$, if $\delta>0$, and to the objective survival probability $\pi(m \mid j)$ if $\delta=0$.

Thus, whenever there is some ambiguity involved, i.e., $\delta>0$, Bayesian learning with psychological bias implies that the impact of the additive part on the overall belief will decrease with increasing age. In the context of survival expectations this allows us to formally express the idea that individuals minimize their "cognitive encounters with death" (Kastenbaum 2000) and suppress the notion of death the more relevant the risk of dying becomes, i.e., the older they are.

\subsection{A Parsimonious Model}

We now develop a simplified version of our model of Bayesian learning with psychological bias that we bring to the data on survival beliefs in section 4 . To this end, we make the following assumptions: First, we restrict ourselves to a linear experience function, i.e., $n(h)=\psi \cdot h$ with $\psi>0$. Second, we assume that the representative agent of age $h$ receives information $I_{h}$ such that $h \subseteq I_{h}$. Under this assumption, the information at a specific age is deterministic rather than random, which can be justified by the fact that the representative agent's information represents the average information of a large population. Third, we suppose that the representative agent observes at every age sample means that actually coincide with the objective survival rates, i.e., $\mu_{n(h)}(m)=\pi(m)$ and $\mu_{n(h)}(j)=\pi(j)$ for all $h$. Again, this assumption is appealing for large populations. Fourth, our data is organized such that interview age $j$ and information $h$ are identical, hence $n(h)=n(j)=\psi \cdot j$ and so forth. Finally, at age $j=0$, we initialize $\tilde{\pi}_{0}(r+1 \mid r)$ for all $r=0, \ldots, m-1$ as

$$
0 \leq \tilde{\pi}_{0}(r+1 \mid r)=\phi \pi(r+1 \mid r) \leq 1
$$

The unconditional belief to survive until interview age $j$ formed at age $0, \tilde{\pi}(j)$, is accordingly given by

$$
\tilde{\pi}(j)=\phi^{j} \pi(j)=\prod_{r=0}^{j-1} \phi \pi(r+1 \mid r),
$$


and we also have that

$$
\tilde{\pi}(m \mid j)=\phi^{m-j} \cdot \pi(m \mid j) .
$$

Using these assumptions in proposition 1 we can summarize our parameterized model of subjective life expectancy as follows:

Proposition 2. The posterior belief of an agent of age $j$ to survive until age $m$ conditional on being alive at age $j$ is

$$
\nu_{j}(m \mid j)=\delta_{j} \cdot \lambda+\left(1-\delta_{j}\right) \cdot \tilde{\pi}_{j}(m \mid j)
$$

such that

$$
\delta_{j}=\frac{\delta}{\delta+(1-\delta) \cdot \phi^{j} \pi(j)}
$$

and

$$
\tilde{\pi}_{j}(m \mid j)=\left(\frac{\phi^{m}+\xi j}{\phi^{j}+\xi j}\right) \cdot \pi(m \mid j)
$$

where

$$
\xi=\frac{\psi}{\alpha+\beta}
$$

Consequently, our simplified version of Bayesian learning with psychological bias results in a parsimonious specification of the representative agent's age-belief pattern with a vector of four parameters, $\Psi=[\phi, \xi, \delta, \lambda]$, only. These parameters reflect (i) an initial bias in the additive estimator reflecting overestimation, i.e., $\phi>1$, or underestimation, i.e., $\phi<1$, (ii) the strength of the rational Bayesian learning process $(\xi)$, (iii) a measure for ambiguity $(\delta$ ), and (iv) the degree of optimism, respectively pessimism, by which the agent resolves her ambiguity $(\lambda)$.

Remark. If there is no ambiguity in the agent's beliefs, i.e., $\delta=0$, our model reduces to a version of rational Bayesian learning by which the agent's subjective belief $\tilde{\pi}_{j}(m \mid j)$ converges to the objective probability $\pi(m \mid j)$ when her actual age $j$ approaches the target age $m$. Depending on an initial overestimation, i.e., $\phi>1$, resp. underestimation, i.e., $\phi<1$, the subjective beliefs thereby monotonically converge from "above", resp. "below", whereby this convergence behavior is the same for all target ages. As already discussed in the introduction, such a model of rational Bayesian learning can obviously not accommodate the stylized facts of figure 1, showing strong underestimation for a lower target age, e.g., $m=80$, and strong overestimation for a higher target age, e.g., $m=95$. In order to accommodate these stylized facts by rational Bayesian learning alone, an according model would require target-age specific parameters $\phi_{m}$ such that, e.g., $\phi_{85}<1$ and $\phi_{95}>1$. Such an extension would come at the cost of loosing parsimony without offering a straightforward interpretation of the additional parameters. In our opinion, it is therefore highly implausible that the HRS data may reflect rational Bayesian learning alone. 


\section{The Data}

According to our model three different types of data are required for the empirical analysis that follows in section 4: (i) subjective conditional beliefs to live until target age, (ii) objective unconditional probabilities to live until interview age and (iii) predicted objective conditional probabilities to live from age $r$ to age $r+1$ for all $r=j, \ldots, m-1$. We here describe our data sources and the methodologies we apply to construct these data.

\subsection{The Data on Subjective Survival Beliefs}

In the HRS, respondents of waves 5 through 7 were asked in the respective interview years $\tau \in\{2000,2002,2004\}$ about their probability to live from interview age $j$ until a certain target age $m$. Recall that target ages are assigned to the respondents' age at interview according to the pattern in table 1.

The HRS is a national representative panel survey of individuals aged 50 and older and their spouses. In addition to respondents from eligible birth years, the survey interviewed the spouses or partners of the respondents, regardless of age. Thus, some (mostly female) individuals are younger than 50 and few, younger than 40. In our application we focus on the target group of the HRS and therefore only look at individuals of age 50 and older. Some respondents of the above question were 90 years old at the time of interview. We do not include these observations in our analysis.

Younger HRS interviewees were also asked about their probabilities to live until age 75 . Some of these respondents have given inconsistent answers at certain points of time as their self-reported probabilities to live until 75 are lower than the selfreported probabilities to live until 80 or 85 . We excluded these cases of evidently inconsistent answering patterns. Furthermore, in some cases, individuals reported the same probability to live until age 75 as to live until age 80 or 85 . As this answering pattern may be due to pure rounding and is not strictly inconsistent with our theoretical model, we keep these observations in the sample.

This selection by age and consistency of answering patterns leaves us with a total sample size of 44671 observations out of which 18341 are male and 26330 are female respondents. We refer to this sample as our "full sample" below.

An apparently more serious problem than such inconsistencies is the existence of "focal point answers" at self-reported survival probabilities of 0, 50, and 100 percent (Hurd and Kathleen 1995; Gan, Hurd, and McFadden 2003). One interpretation for individuals indicating probabilities of 0 or 100 percent is that they have not fully understood the question. ${ }^{10}$ Thus, focal point answers could be regarded as implausible estimates of subjective probabilities. However, as discussed by Smith, Taylor, Sloan, Johnson, and Desvouges (2001) and Khwaja, Sloan, and Chung (2006), these focal

\footnotetext{
${ }^{10} \mathrm{An}$ alternative interpretation is that focal point answers reflect ambiguity, cf. Hill, Perry, and Willis (2004).
} 
point answers at $0 \%$ and $100 \%$ still have information content regarding the correct subjective belief because smokers provide the answer $0 \%$ more frequently than nonsmokers. The target age-group specific answer pattern in our data displayed in figure 3 also illustrates that focal point answers have information content for the true subjective belief because the frequency of focal point answers at $0 \%$ increases with target age whereas the frequency of focal point answers at $100 \%$ decreases with target age. The overall pattern is the same for male and female respondents. Focal point answers at $50 \%$ may be due to round-off (Börsch-Supan 1998) or may reflect that respondents simply do not know much about their individual survival probability (Hurd, Rohwedder, and Winter 2005).

One approach to deal with these problems followed in the literature is to formally correct for focal point answers. Along this line, Gan, Hurd, and McFadden (2003) suggest a Bayesian procedure that reduces the distance between subjective survival curves and observed survival. ${ }^{11}$ In our context, this approach is obviously meaningless because our aim is to explain the difference between subjective beliefs and the objective data. The alternative, followed by Smith, Taylor, and Sloan (2001), Smith, Taylor, Sloan, Johnson, and Desvouges (2001) and Khwaja, Sloan, and Chung (2006), is to acknowledge the information content of focal point answers and to examine the sensitivity of results with respect to these observations. We follow this latter approach.

In our model, focal point answers induce two sorts of biases. On the one hand, focal point answers at $0 \%$ and $100 \%$ bias the degree of pessimism observed at young ages and the degree of optimism observed at older ages, compare figure 1, downward and thereby towards the objective data. This is so because the focal point answer at $100 \%$ is primarily given by younger respondents whereas the focal point answer at $0 \%$ is primarily given by older respondents, compare figure 3. On the other hand, focal point answers at 50\% induce opposite biases towards pessimism at younger ages and towards optimism at older ages. This is so because the objective survival probabilities of younger respondents are above $50 \%$ whereas those of older respondents are below $50 \%$. Consequently, the first form of bias favors our interpretation of the data whereas the second form works against it.

Below, we first present results without any additional corrections to our data (our full sample) and then examine the sensitivity of our results using a sample in which all observations with focal point answers are excluded, cf. figure 4. This correction by focal point answers leaves us with a sample size of 24225 observations (10188 male and 14037 female respondents) out of the aforementioned 44671 observations, that is, roughly $46 \%$ of interviewees have given focal point answers at either $0 \%, 50 \%$ or $100 \%$, respectively.

\footnotetext{
${ }^{11}$ Other researchers, such as Bloom, Canning, Moore, and Song (2006), correct for focal point answers by instrumental variables techniques.
} 
Figure 3: Answer pattern

(a) Men

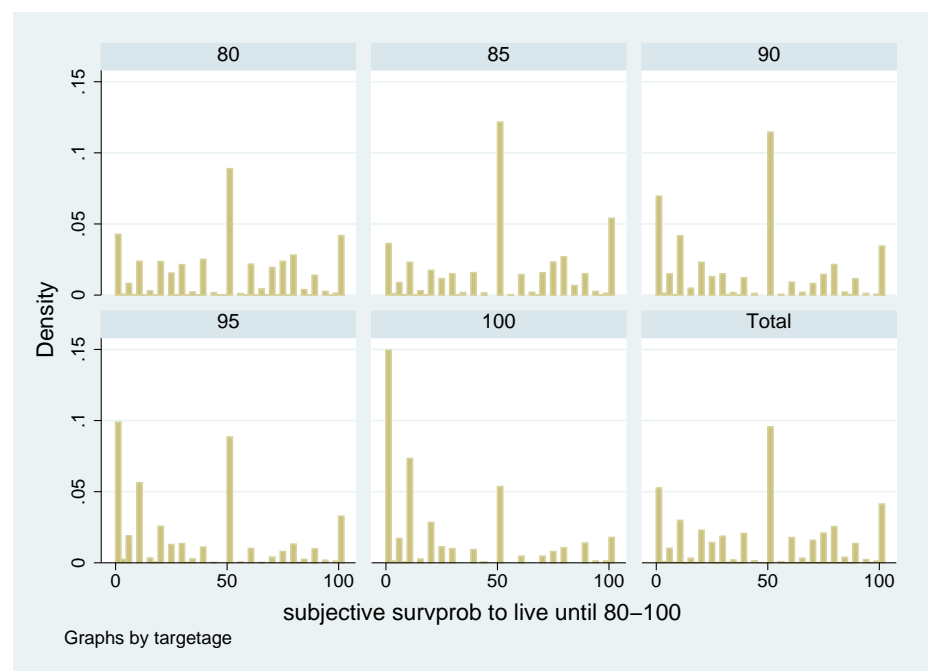

(b) Women

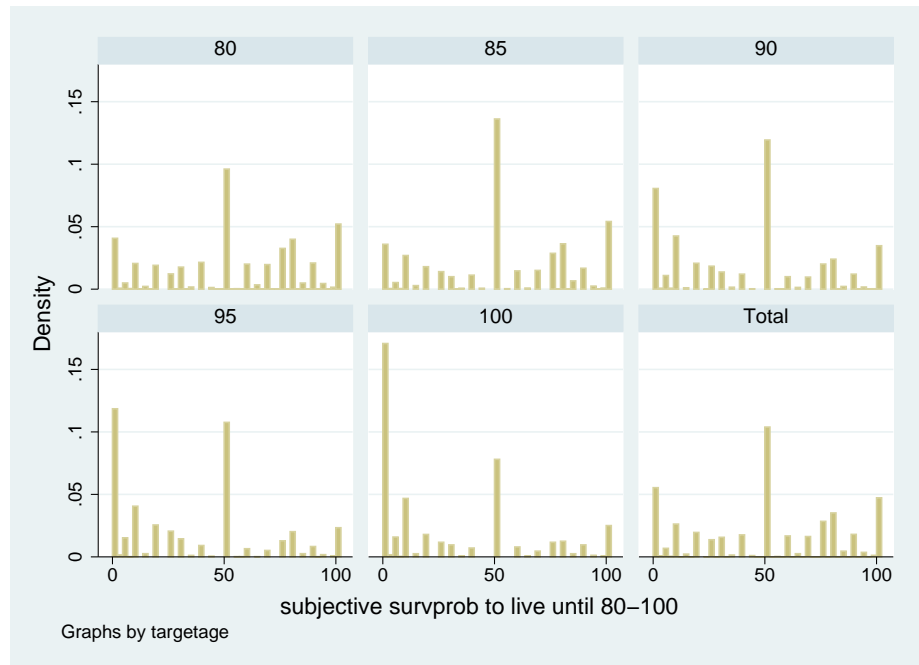

Source: Own calculations based on HRS data. 
Figure 4: Answer pattern (excluding focal point answers)

(a) Men

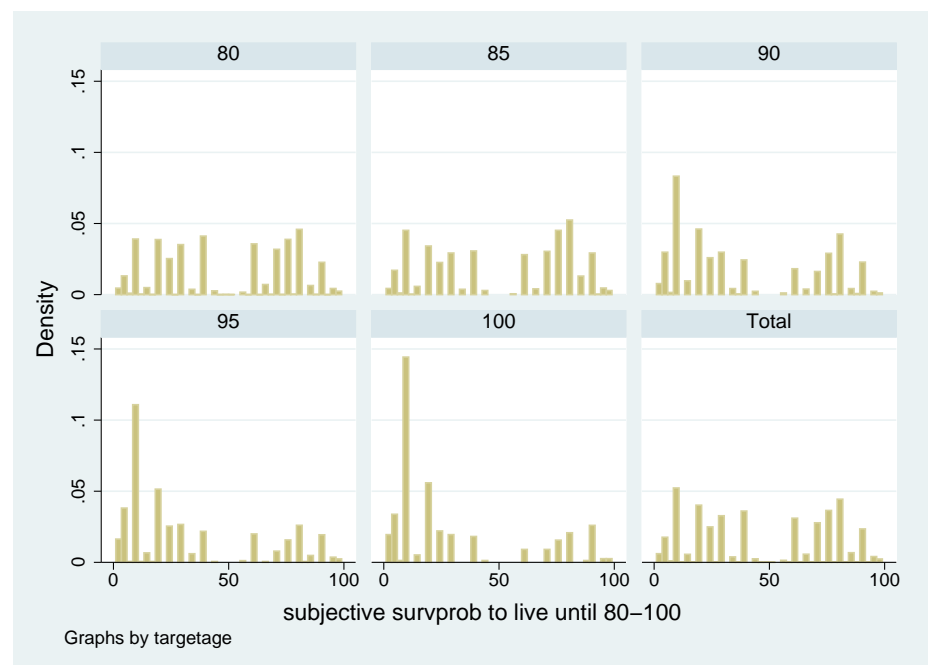

(b) Women

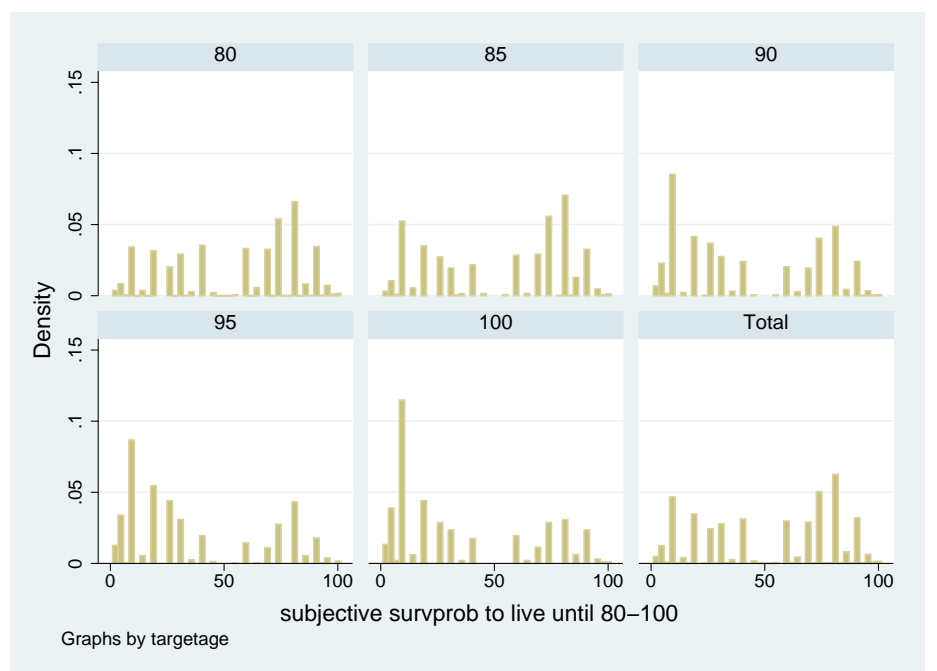

Source: Own calculations based on HRS data. 


\subsection{Objective survival probabilities to live until interview age}

According to proposition $2, \delta_{j}$ is a function of the initial degree of ambiguity, $\delta$, and the subjective probability $\tilde{\pi}(j)$ to survive until interview age $j$. By our assumption in equation (6), the latter is a function of the true objective probability to reach age $j, \pi(j)$. We calculate $\pi(j)$ from cohort life tables taken from the Human Mortality Database (HMD) and the Social Security Administration (SSA).

\subsection{Predicted objective survival probabilities}

According to proposition $2, \nu(m \mid j)$ is a weighted average of the degree of optimism $\lambda$, and the subjective conditional probability to live from interview age $j$ to target age $m, \tilde{\pi}(m \mid j)$. By equation (6), the latter is a function of the true objective conditional probabilities to reach age $r+1$ conditional on age $r$ in the future for $r=j, \ldots, m-1$. To calculate the predicted values $\pi(r+1 \mid r)$ we predict future survival rates in the population. Our estimates are based on data for age-specific survival rates for the years 1900 to 2004 taken from the HMD and the SSA. Since projections from official sources tend to underestimate future increases in survival probabilities, we do not use SSA cohort life tables but rather base the prediction of future survival rates on a Lee-Carter (Lee and Carter 1992) procedure. The idea of our approach is that the agents in our model base their predictions of their respective objective survival probabilities on past data but it is unobserved to the econometrician which point estimates they use. For this reason we account for the uncertainty of the objective data in the estimation. As an additional advantage, our procedure assigns the objective information on survival rates in correspondence with the HRS interview years, see below. As a sensitivity analysis, we also report estimation results in appendix A.3 which are directly based on the SSA cohort file tables.

The Lee-Carter procedure allows us to describe and to project the development of age-specific mortality rates over time within a parsimonious framework. Basically, the model splits mortality rates into age-specific components that are constant over time and a time varying survival index capturing the development of mortality. Then, one can extrapolate the time series of the mortality index by means of a suitable time series model. Future age-specific mortality rates can be recovered by linking the projected mortality index to the age-specific components.

To describe the methodology, we now introduce a time index $t$. Following Lee and Carter (1992) we decompose the average objective age-specific survival probability in period $t$ as

$$
\log \left(\pi_{t}(r+1 \mid r)\right)=a_{r}+b_{r} d_{t}
$$

where $a_{r}$ and $b_{r}$ are the age-specific constants and where $d_{t}$ is the time specific factor. We opt for a parsimonious representation of the time series process of $d_{t}$ and assume that $d_{t}$ follows a unit root process with drift

$$
d_{t}=\theta+d_{t-1}+\epsilon_{t}
$$


where $\epsilon \sim \mathcal{N}\left(0, \sigma_{\epsilon}^{2}\right)$.

We assign objective survival probabilities to each respondent in our HRS panel in each wave $\tau \in\{2000,2002,2004\}$ as follows. We estimate for each wave $\tau$, sex specific values of $\hat{a}_{j}, \hat{b}_{j}, \hat{\theta}, \hat{\sigma}_{\epsilon}$ and calculate predicted values of $\hat{\pi}_{t}(r+1 \mid r)$ using data only until period $\tau$. We then proceed to the next wave and update the objective information also using the data for the two years in between periods $\tau$ and $\tau+2$. Our predictions of future objective survival probabilities, $\hat{\pi}_{t}(r+1 \mid r)$, are calculated by iterating forward on

$$
\hat{d}_{t}=\hat{\theta}+\hat{d}_{t-1}
$$

and

$$
\hat{\pi}_{t}(r+1 \mid r)=\exp \left(\hat{a}_{r}+\hat{b}_{r} \hat{k}_{t}\right) .
$$

While we ignore uncertainty of our estimates of the age-vectors $a_{r}$ and $b_{r}$, we account for uncertainty of the objective data by calculating standard deviations and confidence intervals of $\hat{\theta}$ by bootstrapping. Table 4 in appendix A.2 contains the relevant information on the sex and wave specific point estimates of $\hat{\theta}$ and the respective standard deviations.

Figure 5 shows data on, and predicted values for, the remaining life expectancy at age 65 for wave 2002. The dashed lines are the bootstrapped $95 \%$ confidence intervals. The new information on objective survival probabilities between waves only leads to small changes in these predictions. Results for other years are therefore not shown. Furthermore, life expectancy at birth and the remaining life-expectancies at other ages display similar trends whereby the trend is increasing with age.

\subsection{Selectivity}

One criticism raised against using population averages as the relevant objective data is that our HRS sample may be prone to selectivity. Reasons for such selection biases are either that households have moved to nursing homes and are not followed by HRS interviewers or that sick people are reachable but may not be able to answer the questionnaire. ${ }^{12}$ Such selection effects may explain (some of) the optimism we observe at higher ages in figure 1.

To address these concerns, we compute the HRS hazard rates between waves 2000 and 2002 and between waves 2002 and 2004, respectively, and compare them to the biannual mortality rates in the population for the respective years. In figure 6 we display the resulting hazard rates for men in panel (a) and for women in panel (b) between waves 2002 and 2004 for our full sample. The wiggles in the HRS data (dashed lines) are a consequence of the small sample size. Evidently, the HRS hazard rates correspond with the mortality rates in the population. The pattern is similar for

\footnotetext{
${ }^{12}$ As Mike Hurd pointed out to us, the first selection effect was particularly severe for the early waves of the HRS and may, if at all, only play a role for the very old respondents in our sample.
} 
Figure 5: Predicted life expectancy at age 65 in year 2002

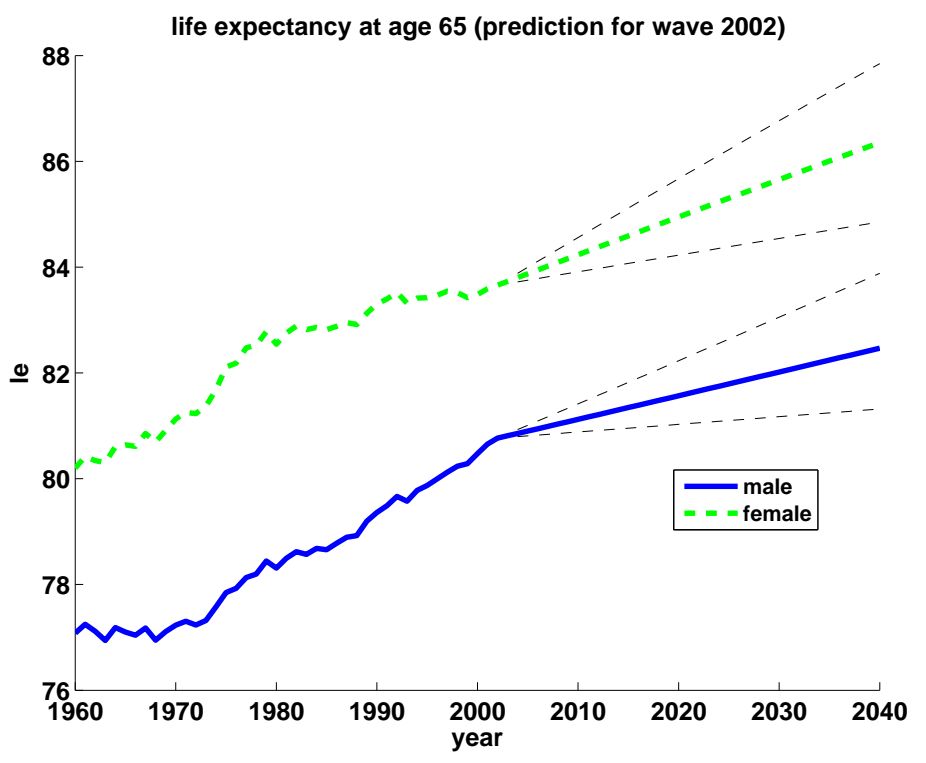

Notes: Black dashed lines are $95 \%$ confidence intervals obtained from 500 bootstrap iterations.

Source: Own calculations based on HMD and SSA data.

the hazard rates between waves 2000 and 2002 (and also for our sample corrected by focal point answers) and therefore not shown. For ages above 75 we even found slightly higher mortality rates in the HRS between waves 2000 and 2002 than in the population which gives even more support to our interpretation of the data as "optimism" at higher ages.

\section{Empirical Results}

\subsection{Estimation Strategy}

We estimate the model separately for men and women but again, for notational convenience, we do not display an index for sex. To estimate the four parameters of our model, $\Psi=[\phi, \xi, \delta, \lambda]$, we pool a sample of the HRS data formed of the HRS waves $\{2000,2002,2004\}$. In each interview age group $j$ we then have $N_{j}$ observations denoted as $i \in\left\{1, \ldots, N_{j}\right\}$ where $N_{j}$ differs across groups. In our estimation we weigh observations by the inverse of the group sizes, $\frac{1}{N_{j}}$, such that we down-weigh age groups with many observations relative to age groups with few observations and vice versa. ${ }^{13}$

\footnotetext{
${ }^{13}$ Observe that this weighting scheme implies that our point estimates are identical to a regression based on the average survival rates in each group. Parameter estimates from an un-weighted regression are similar and are available upon request.
} 
Figure 6: Objective survival rates in 2002-2003: HRS data versus population averages

(a) Men

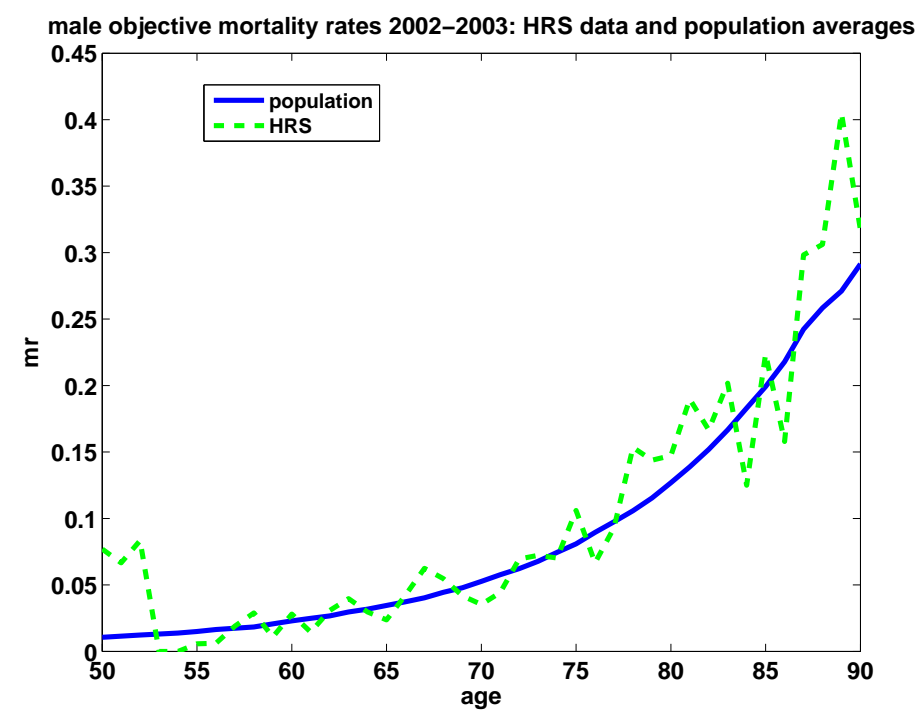

(b) Women

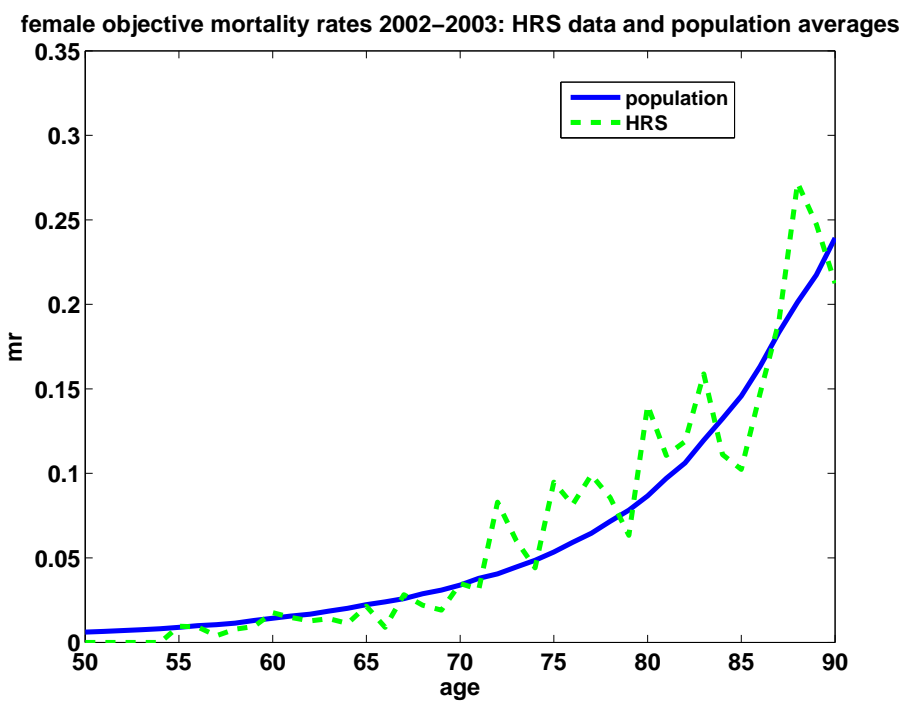

Notes: Solid line: population wide hazard rates (mortality rates) for 2002-2003. Dashed line: HRS hazard rates (mortality rates) between waves 2002 and 2004.

Source: Own calculations based on HRS, SSA and HMD data. 
To determine the parameter values we accordingly solve the following non-linear minimization problem

$$
\min _{\Psi} \frac{1}{2} \sum_{j=1}^{J} \frac{1}{N_{j}} \sum_{i=1}^{N_{j}}\left(\nu_{i}(m \mid j)-\hat{\nu}(m \mid j)\right)^{2}
$$

Here, $\nu_{i}(m \mid j)$ denotes individual $i$ 's conditional subjective belief to survive from interview age $j$ to target age $m$ in the HRS data, whereby target ages are assigned to interview ages according to the pattern in table $1 . \hat{\nu}(m \mid j)$ is the predicted subjective belief according to our model as described in proposition 2 .

We solve the above non-linear programming problem subject to the inequality constraints

$$
\begin{aligned}
& \delta \in[0,1], \quad \lambda \in[0,1], \quad \xi \in[0,1] \text { and } \\
& 0 \leq \phi \cdot \pi(r+1 \mid r) \leq 1 \text { for all } r=0, \ldots, m-1
\end{aligned}
$$

using a constrained optimizer. As unique convergence is not guaranteed for such problems, we tried various combinations of starting values, $\Psi_{0}$, for all of our scenarios that follow. For all these combinations the solver returned the same solution vector $\hat{\Psi}$. We are therefore confident that the solver converges to the unique global minimum. We bootstrap standard errors by drawing with replacement from our data on subjective beliefs and from our predicted data on objective survival probabilities in a total of 500 bootstrap iterations. With one exception, see section 4.3 below, the bound constraints on the parameters in equation (13) were never binding in all these iterations.

\subsection{Main Results}

Our main estimation results, based on the sample including all observations with focal point answers, compare figure 3, are summarized in table 2. The upper part of the table shows parameter estimates in the case of non-ambiguous beliefs resulting in the standard model of rational Bayesian learning. The lower part of the table shows the respective estimates for our preferred specification with ambiguous beliefs where, in addition to rational Bayesian learning, the learning process may be prone to psychological bias. For each estimated parameter, the table contains sex specific information on the point estimates, $\hat{\Psi}$, the respective standard errors, $\hat{\sigma}(\Psi)$, and the $95 \%$ confidence intervals of the coefficient estimates, $\widehat{C I}(\psi)$. In order to document the fraction of the overall variation of survival probabilities in the data that is explained by the respective parsimonious model we also report the $R^{2}$ of the regressions. In addition, we report an "average $R^{2}$ ", denoted as $\bar{R}^{2}$, as a measure of the fraction of the variation in average survival probabilities explained by our model.

From the table entries we already observe that the model featuring only rational Bayesian learning is rejected because it does not have explanatory power in the case 
Table 2: Parameter estimates

\begin{tabular}{lccc|ccc}
\hline \hline & \multicolumn{6}{c}{ Rational Bayesian learning } \\
\hline & \multicolumn{4}{c}{ Men } & \multicolumn{4}{c}{ Women } \\
\hline & $\hat{\Psi}$ & $\hat{\sigma}(\Psi)$ & $\widehat{C I}(\psi)$ & $\hat{\Psi}$ & $\hat{\sigma}(\Psi)$ & $\widehat{C I}(\psi)$ \\
\hline Initial pessimism: $\phi$ & 0.990 & 0.002 & {$[0.987,0.994]$} & 0.986 & 0.001 & {$[0.985,0.988]$} \\
Update parameter: $\xi$ & 0.067 & 0.021 & {$[0.039,0.111]$} & 0.006 & 0.002 & {$[0.002,0.011]$} \\
\hline$R^{2}$ & -0.013 & 0.011 & {$[-0.038,0.006]$} & 0.030 & 0.006 & {$[0.016,0.041]$} \\
$\bar{R}^{2}$ & -0.393 & 0.226 & {$[-0.863,0.023]$} & 0.366 & 0.098 & {$[0.141,0.524]$} \\
\hline \hline \multicolumn{6}{c}{ Psychologically biased Bayesian learning } \\
\hline \multicolumn{6}{c}{$\hat{c}$ Men } & \multicolumn{4}{c}{ Women } \\
\hline Initial pessimism: $\phi$ & 0.991 & 0.002 & {$[0.987,0.995]$} & 0.993 & 0.001 & {$[0.991,0.995]$} \\
Update parameter: $\xi$ & 0.037 & 0.016 & {$[0.017,0.081]$} & 0.005 & 0.005 & {$[-0.005,0.016]$} \\
Degree of ambiguity: $\delta$ & 0.173 & 0.033 & {$[0.121,0.245]$} & 0.321 & 0.022 & {$[0.280,0.369]$} \\
Degree of optimism: $\lambda$ & 0.481 & 0.026 & {$[0.433,0.533]$} & 0.397 & 0.018 & {$[0.368,0.430]$} \\
\hline$R^{2}$ & 0.041 & 0.004 & {$[0.032,0.048]$} & 0.066 & 0.003 & {$[0.060,0.071]$} \\
$\bar{R}^{2}$ & 0.784 & 0.037 & {$[0.677,0.820]$} & 0.960 & 0.010 & {$[0.923,0.962]$} \\
\hline \hline
\end{tabular}

Notes: $\hat{\Psi}$ are point estimates of model parameters, $\hat{\sigma}(\Psi)$ is the respective standard deviation and $\widehat{C I}(\Psi)$ is the respective $95 \%$ confidence interval. Standard errors are calculated by bootstrapping the subjective and objective survival probabilities by drawing with replacement in 500 bootstrap iterations.

Source: Own calculations based on HRS, SSA and HMD data. 
of men (the $R^{2}$ is negative and insignificant). ${ }^{14}$ Figure 7 illustrates why the model with non-ambiguous beliefs is performing so poorly by comparing the subjective data to the predicted values from the model. First, observe that there is a strong initial underestimation of life-expectancy which is reflected in the precise estimates of $\phi<1$ for both men and women. To interpret the (precise) point estimates of this parameter, i.e., $\phi=0.990$ for men and $\phi=0.986$ for women, observe that $\phi^{30}$ captures the initial underestimation of an agent of interview age 50 to survive until target age 80, cf. equation (7). In the data we observe an underestimation by -13.70 percentage points for men ( -15.07 percentage points for women) and our point estimates of $\phi$ imply -13.67 percentage points for men and -23.35 for women. While slightly overestimating the initial pessimism of women, the model therefore broadly captures the initial biases observed in the data. Second, as already discussed in section 1, we observe only a relatively low degree of convergence of the subjective data to the objective data at young ages. Consequently, the point estimates of $\xi$ are generally low and numerically even close to zero in the case of women. Third, rational Bayesian learning implies convergence of subjective beliefs to objective probabilities for high ages, cf. observation 1. As the subjective data are above the objective data at higher ages, the rational Bayesian updating model therefore fails to match the actual patterns of subjective beliefs.

Quite in contrast, our model of psychologically biased Bayesian learning which considers ambiguous beliefs results in a decent fit to the average subjective survival expectations, compare figure 8 . For both men and women, predicted subjective beliefs track the average subjective beliefs from the data nicely. An exception is the fit to survival expectations at higher ages for men. Here, psychologically biased Bayesian learning converges faster to $\lambda$ than for women because convergence of $\delta_{j}$ to 1 is faster, cf. figure 9. This faster convergence is caused by the lower objective unconditional survival probabilities of men to reach interview age $j$, cf. proposition 2. Consequently, the $R^{2}$ of the regressions is significantly higher for women than for men, compare table $2 .{ }^{15}$ Unsurprisingly, our parsimonious specification of average beliefs results in low $R^{2} \mathrm{~s}$ of the regressions - 0.041 for men and 0.066 for women - because the model can only capture some of the variation in answer patterns. The model however explains 78.4 percent of the average variation of subjective beliefs of men (95.9 percent in the case of women).

As far as the point estimates are concerned we observe that the changes in the point estimates of $\phi$, the initial pessimism, are low when we move from the rational Bayesian learning to the psychologically biased Bayesian learning model. Coming back

\footnotetext{
${ }^{14}$ We calculate the $R^{2}$ as $1-R S S / T S S$, where $R S S$ is the residual sum of squares and $T S S$ is the total sum of squares. It is well-known that $R^{2}$ calculated as such is not guaranteed to be in the range of $[0,1]$ in non-linear regression models, cf. Greene (2008, Ch. 11).

${ }^{15}$ The value of the two-sided $t$-test on the difference between the $R^{2}$ s for men and women is 108.77 with a $p$-value of 0.0 . The values of Jarque-Bera test statistics for normality of the distribution of the bootstrapped $R^{2} \mathrm{~s}$ (and their $p$-values) are at $2.18(0.33)$ for men and at $4.48(0.11)$ for women such that a standard $t$-test is applicable.
} 
Figure 7: Actual and predicted survival probabilities for rational Bayesian learning

(a) Men

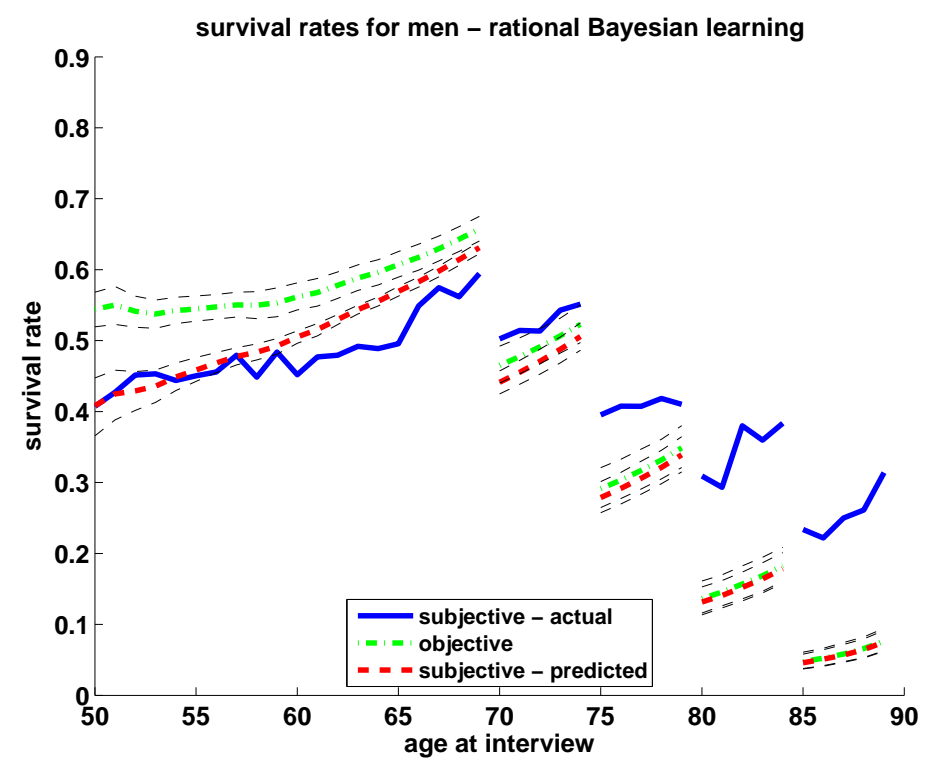

(b) Women

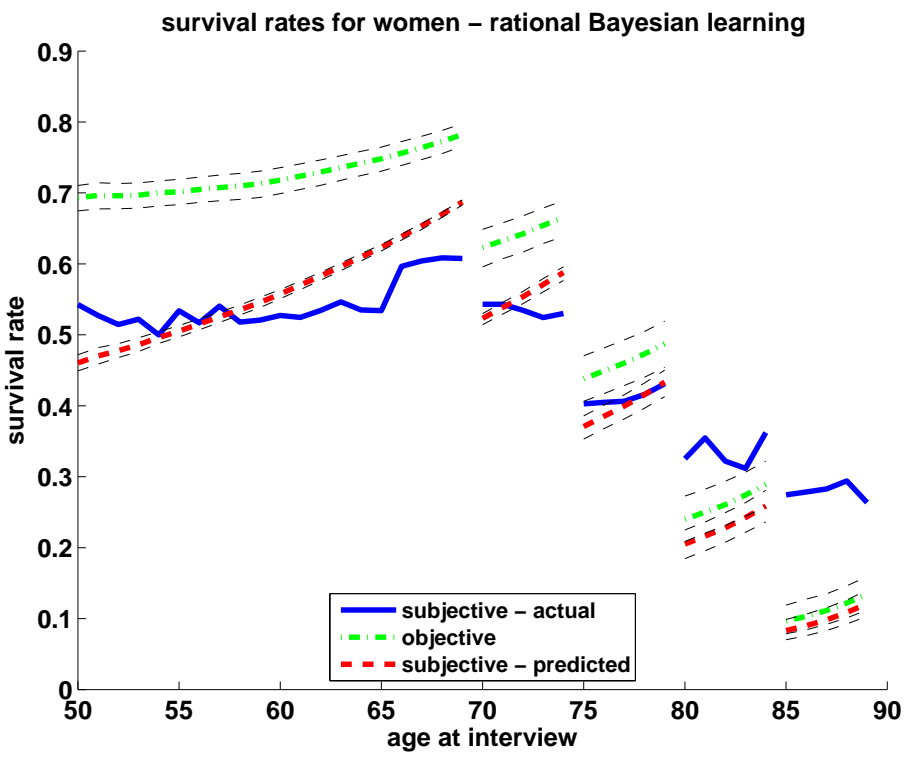

Notes: Black dashed lines are $95 \%$ confidence intervals obtained from 500 bootstrap iterations. Source: Own calculations based on HRS, HMD and SSA data. 
to our example of the subjective belief of a 50 year old to reach target age 80 , the estimated values of $\phi$ now imply an underestimation by -12.80 percentage points for men $(-13.34$ precentage points for women) compared to -13.70 percentage points $(-15.07$ percentage points) in the data. As part of the rational Bayesian learning motive at work in our model is now superseded by psychologically biased learning, the point estimates of $\xi$ for men are significantly lower than before and for women the estimated parameter value is now insignificant.

We further find that the measure of optimism under ambiguity is significantly higher for men, i.e., $\lambda=0.481$ with a $95 \%$ confidence interval of [0.433, 0.533], than for women, i.e., $\lambda=0.397$ with a $95 \%$ confidence interval of $[0.368,0.430]$, and that the estimated ambiguity is initially significantly lower for men, i.e., $\delta=0.173$ with a $95 \%$ confidence interval of $[0.121,0.245]$, than for women, i.e., $\delta=0.321$ with a $95 \%$ confidence interval of $[0.280,0.369]$. According to our interpretation of ambiguous beliefs, the weight $\left(1-\delta_{j}\right)$ measures how much evidence gained from rational Bayesian learning is taken into account. Conversely, $\delta_{j}$ corresponds to the weight by which beliefs are affected by some "myside bias," in our model formalized as personal attitudes towards optimism, resp. pessimism, as measured by $\lambda$. A literal interpretation of our estimation results would therefore suggest that, on the one hand, the belief formation of men initially starts out more rational than that of women in that men put more weight on the available evidence. However, as already discussed above, $\delta_{j}$ is found to increase more strongly for men than for women with increasing age $j$, cf. figure 9 . Thus, on the other hand, men are apparently more prone to an irrational "myside bias" than women in the long run whereby men are more optimistically biased than women.

Because of the simplicity of our model - owed to our wish for parsimony - we do not want to overstretch the significance of such specific interpretations. As a very robust result of our analysis, however, we find for both genders that the assumption of a psychological "myside bias" in the interpretation of new information can better explain the survival belief formation of a representative agent than the assumption of a learning process which is based on evidence alone.

\subsection{Sensitivity Analysis - Focal Point Answers}

As discussed in section 3, our analysis may be confounded by the presence of focal point answers. We therefore repeat our estimation using a second data set where all observations with focal point answers are excluded. Estimation results for this alternative data set are summarized in table 3. A comparison with our benchmark results in table 2 shows that the broad pattern of estimated values does not change much. We here report results from a constrained model with $\xi=0$ in the case of women because the point estimate of $\xi$ was negative for these data and again find that the (constrained) model performs statistically better for women. ${ }^{16}$

\footnotetext{
${ }^{16}$ The value of the two-sided $t$-test is 20.24 with a $p$-value of 0.0 . The values of Jarque-Bera test statistics for normality of the distribution of the bootstrapped $R^{2}$ s (and their $p$-values) are at 2.15
} 
Figure 8: Actual and predicted survival probabilities for psychologically biased Bayesian learning
(a) Men

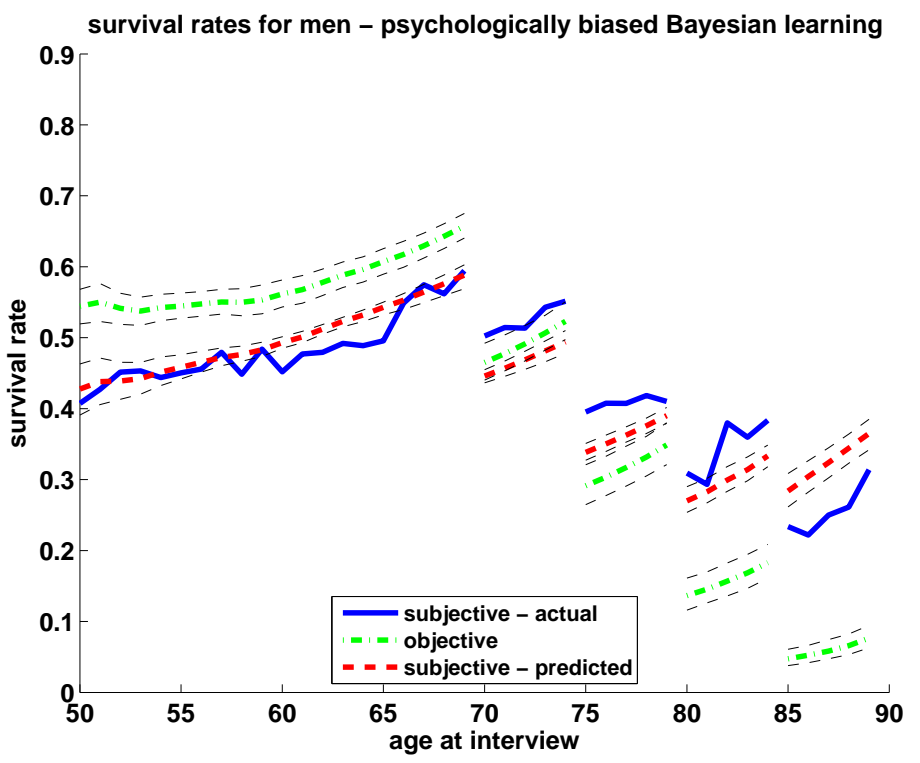

(b) Women

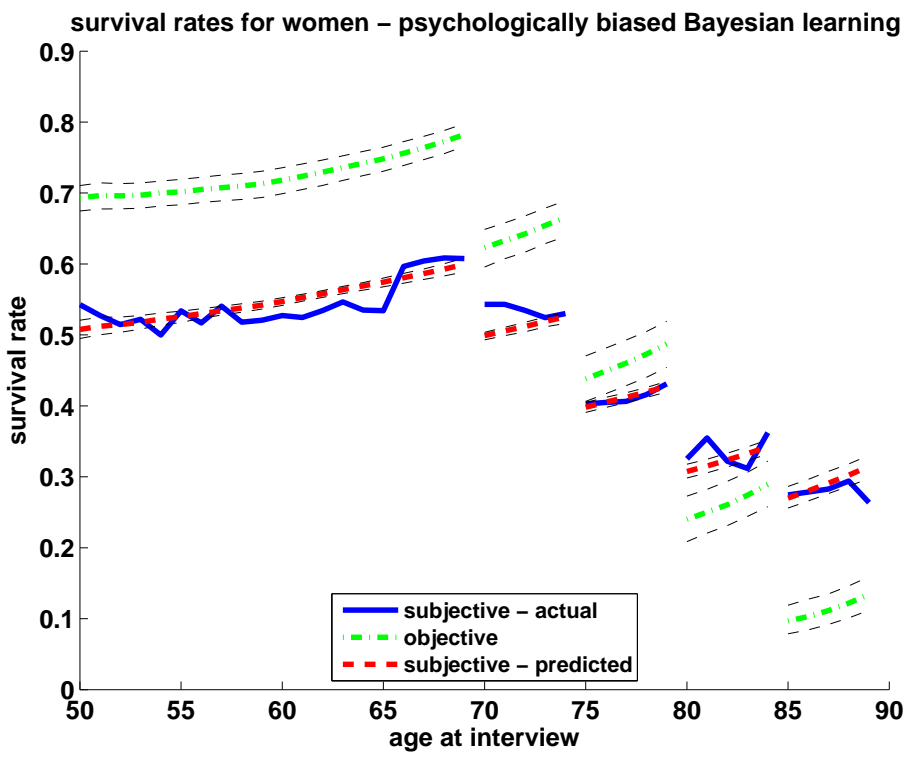

Notes: Black dashed lines are $95 \%$ confidence intervals obtained from 500 bootstrap iterations. Source: Own calculations based on HRS, HMD and SSA data. 
Figure 9: Degree of ambiguity $\left(\delta_{j}\right)$

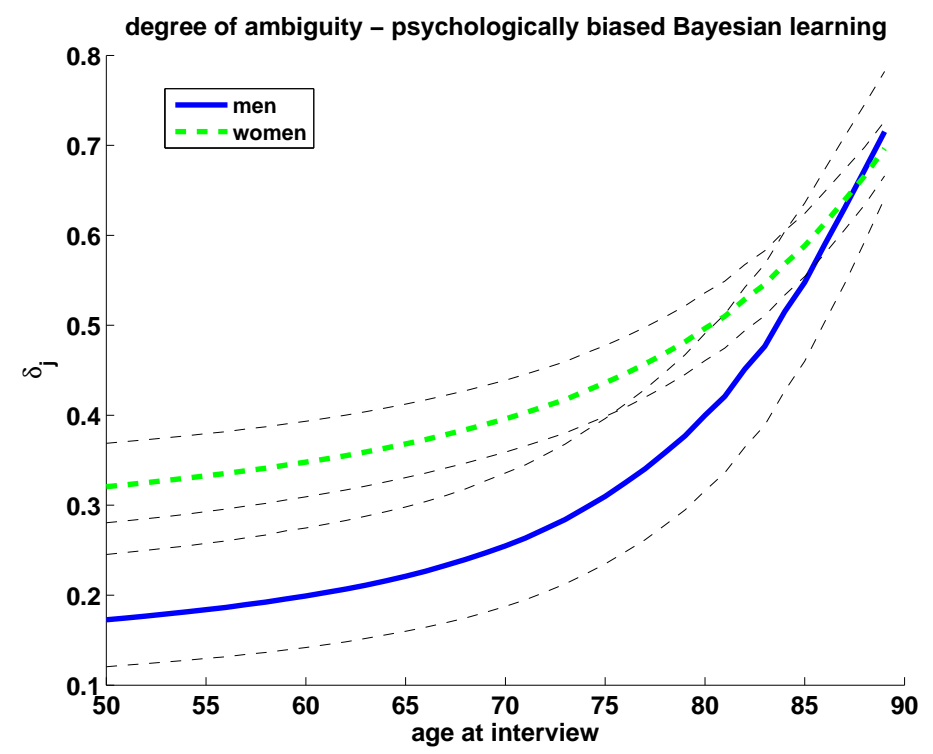

Notes: Black dashed lines are $95 \%$ confidence intervals obtained from 500 bootstrap iterations. Source: Own calculations based on HRS, HMD and SSA data.

Table 3: Parameter estimates (excluding focal point answers)

\begin{tabular}{lccc|ccc}
\hline \hline & \multicolumn{4}{c}{ Psychologically biased Bayesian learning } \\
\hline & \multicolumn{4}{c|}{ Men } & \multicolumn{4}{c}{ Women } \\
\hline & $\hat{\Psi}$ & $\hat{\sigma}(\Psi)$ & $\widehat{C I}(\psi)$ & $\hat{\Psi}$ & $\hat{\sigma}(\Psi)$ & $\widehat{C I}(\psi)$ \\
\hline Initial pessimism: $\phi$ & 0.992 & 0.003 & {$[0.987,0.997]$} & 0.995 & 0.001 & {$[0.993,0.999]$} \\
Update parameter: $\xi$ & 0.021 & 0.082 & {$[0.001,0.137]$} & 0 & - & - \\
Degree of ambiguity: $\delta$ & 0.208 & 0.046 & {$[0.127,0.317]$} & 0.436 & 0.030 & {$[0.384,0.503]$} \\
Degree of optimism: $\lambda$ & 0.460 & 0.044 & {$[0.408,0.536]$} & 0.429 & 0.014 & {$[0.401,0.457]$} \\
\hline$R^{2}$ & 0.045 & 0.005 & {$[0.033,0.053]$} & 0.053 & 0.004 & {$[0.044,0.060]$} \\
$\bar{R}^{2}$ & 0.849 & 0.129 & {$[0.129,0.848]$} & 0.900 & 0.029 & {$[0.803,0.915]$} \\
\hline \hline
\end{tabular}

Notes: $\hat{\Psi}$ are point estimates of model parameters, $\hat{\sigma}(\Psi)$ is the respective standard deviation and $\widehat{C I}(\Psi)$ is the respective $95 \%$ confidence interval. Standard errors are calculated by bootstrapping the subjective and objective survival probabilities by drawing with replacement in 500 bootstrap iterations.

Source: Own calculations based on HRS, SSA and HMD data. 


\section{Conclusion}

The HRS data on subjective survival beliefs suggest a violation of the rational expectations paradigm as well as of the rational Bayesian learning hypothesis. In a first step we therefore propose a new model of Bayesian learning that encompasses rational Bayesian learning while it additionally allows for the existence of a psychological bias in the interpretation of new information. For this purpose our formal approach combines concepts, such as non-additive beliefs and generalized Bayesian updating, from the theory of decision making under ambiguity with the standard approach of rational Bayesian updating. The resulting model of psychologically biased belief formation is very parsimonious in that it requires a low parametrization reflecting, first, an initially biased additive estimator of subjective survival probabilities, second, a measure of the strength of the rational Bayesian updating process, third, a measure for the agent's ambiguity with respect to her initial estimator of her subjective survival probability, and, fourth, a measure for the agent's optimistic versus pessimistic attitudes with respect to this ambiguity.

In a second step we estimate the parameters of our theoretical model by pooling the HRS data. Despite the parsimonious parametrization we find that our model results in a remarkable fit to the average subjective beliefs expressed in the data. The model's performance is statistically better for women than for men. For both genders we can clearly reject the hypothesis that the HRS data on subjective survival probabilities may be explained by rational Bayesian learning. The reason is that the rational Bayesian learning hypothesis implies convergence of the subjective probabilities to the respective objective data at higher ages but we instead observe an increasing degree of optimism in the data. On the contrary, our more sophisticated model of psychologically biased Bayesian learning can match these patterns in the data.

In our model we condition the updating of subjective beliefs only on the age of individuals by which we obtain a representative agent interpretation. The strength of this parsimonious approach is that we can directly map our model into life-cycle models of consumption and savings. In our future research we will use this framework to discuss the demand for annuities and to evaluate the implications of our model for life-cycle consumption and savings profiles. Another avenue of research which we plan to follow is an empirical study in which we condition updating of beliefs on observed idiosyncratic shocks in between waves of the HRS, similar to Smith, Taylor, Sloan, Johnson, and Desvouges (2001).

(0.34) for men and at $1.91(0.38)$ for women such that a standard $t$-test is suitable. 


\section{A Appendix}

\section{A.1 Generalized Bayesian Update Rule}

In the present paper, we consider the so-called generalized (or full) Bayesian update rule. An axiomatic foundation under the assumption of CEU preferences is provided in Eichberger, Grant, and Kelsey (2006).

Definition 2. The generalized Bayesian update rule for determining the conditional capacity $\nu_{I}, I \in \Sigma$, for a given prior capacity $\nu$ is given as follows: for all $E \in \Sigma$,

$$
\nu_{I}(E)=\frac{\nu(E \cap I)}{\nu(E \cap I)+1-\nu(E \cup \neg I)} .
$$

Observation 2. Let $E, I \notin\{\emptyset, S\}$ and $E \cap I \neq \varnothing$. Then an application of the generalized Bayesian update rule to a neo-additive prior results in the posterior belief

$$
\nu_{I}(E)=\delta_{I} \cdot \lambda+\left(1-\delta_{I}\right) \cdot \tilde{\pi}_{I}(E)
$$

such that

$$
\delta_{I}=\frac{\delta}{\delta+(1-\delta) \cdot \tilde{\pi}(I)} .
$$

Proof. Let $E, I \notin\{\emptyset, S\}$ and $E \cap I \neq \varnothing$. Then

$$
\begin{aligned}
\nu_{I}(E) & =\frac{\delta \cdot \lambda+(1-\delta) \cdot \tilde{\pi}(E \cap I)}{\delta \cdot \lambda+(1-\delta) \cdot \tilde{\pi}(E \cap I)+1-(\delta \cdot \lambda+(1-\delta) \cdot \tilde{\pi}(E \cup \neg I))} \\
& =\frac{\delta \cdot \lambda+(1-\delta) \cdot \tilde{\pi}(E \cap I)}{1+(1-\delta) \cdot(\tilde{\pi}(E \cap I)-\tilde{\pi}(E \cup \neg I))} \\
& =\frac{\delta \cdot \lambda+(1-\delta) \cdot \tilde{\pi}(E \cap I)}{1+(1-\delta) \cdot(\tilde{\pi}(E \cap I)-\tilde{\pi}(E)-\tilde{\pi}(\neg I)+\tilde{\pi}(E \cap \neg I))} \\
& =\frac{\delta \cdot \lambda+(1-\delta) \cdot \tilde{\pi}(E \cap I)}{1+(1-\delta) \cdot(-\tilde{\pi}(\neg I))} \\
& =\frac{\delta \cdot \lambda+(1-\delta) \cdot \tilde{\pi}(E \cap I)}{\delta+(1-\delta) \cdot \tilde{\pi}(I)} \\
& =\delta_{I} \cdot \lambda+\left(1-\delta_{I}\right) \cdot \tilde{\pi}_{I}(E)
\end{aligned}
$$

with $\delta_{I}$ given by $(15)$.

\section{A.2 Estimates of Stochastic Trends in Life Expectancy}

Table 4 reports the estimated $\theta$-coefficients of equation (9) of our population model. Estimated parameter values for wave 1,2 and 3 are based on population data from HMD and SSA for 1900 - 2000, 1900 - 2002 and 1900 - 2004, respectively. 
Table 4: Parameter estimates of $\hat{\theta}$

\begin{tabular}{ccc|cc}
\hline \hline & \multicolumn{2}{c|}{ Men } & \multicolumn{2}{c}{ Women } \\
\hline & $\hat{\theta}$ & $\hat{\sigma}(\theta)$ & $\hat{\theta}$ & $\hat{\sigma}(\theta)$ \\
\hline wave 1 & -1.4186 & 0.5336 & -1.8586 & 0.5339 \\
wave 2 & -1.4123 & 0.5426 & -1.8287 & 0.5336 \\
wave 3 & -1.4518 & 0.4927 & -1.8462 & 0.5103 \\
\hline \hline
\end{tabular}

Notes: Standard errors of $\hat{\theta}$ are calculated from 500 bootstrap iterations.

Source: Own calculations based on SSA and HMD.

\section{A.3 Estimation Results for Alternative Objective Data}

We here investigate sensitivity of our results with respect to alternative objective data using the SSA data instead of our own projections. As shown in table 5, results do not differ much compared to our benchmark results in table 2. Standard errors are slightly lower when we use the SSA data because there we do not account for the uncertainty underlying the predictions of the objective survival rates.

Table 5: Parameter estimates (using the predictions of the SSA)

\begin{tabular}{lccc|ccc}
\hline \hline & \multicolumn{4}{c}{ Psychologically biased Bayesian learning } \\
\hline & \multicolumn{4}{c|}{ Men } & \multicolumn{4}{c}{ Women } \\
\hline & $\hat{\Psi}(\Psi)$ & $\widehat{C I}(\psi)$ & $\hat{\Psi}$ & $\hat{\sigma}(\Psi)$ & $\widehat{C I}(\psi)$ \\
\hline Initial pessimism: $\phi$ & 0.993 & 0.002 & {$[0.989,0.996]$} & 0.995 & 0.001 & {$[0.993,0.997]$} \\
Update parameter: $\xi$ & 0.057 & 0.024 & {$[0.031,0.114]$} & 0.005 & 0.006 & {$[-0.006,0.018]$} \\
Degree of ambiguity: $\delta$ & 0.196 & 0.027 & {$[0.148,0.249]$} & 0.309 & 0.020 & {$[0.267,0.346]$} \\
Degree of optimism: $\lambda$ & 0.499 & 0.021 & {$[0.468,0.546]$} & 0.414 & 0.014 & {$[0.395,0.446]$} \\
\hline$R^{2}$ & 0.038 & 0.003 & {$[0.031,0.045]$} & 0.066 & 0.003 & {$[0.060,0.072]$} \\
$\bar{R}^{2}$ & 0.707 & 0.043 & {$[0.616,0.737]$} & 0.962 & 0.010 & {$[0.926,0.963]$} \\
\hline \hline
\end{tabular}

Notes: $\hat{\Psi}$ are point estimates of model parameters, $\hat{\sigma}(\Psi)$ is the respective standard deviation and $\widehat{C I}(\Psi)$ is the respective $95 \%$ confidence interval. Standard errors are calculated by bootstrapping the subjective and objective survival probabilities by drawing with replacement in 500 bootstrap iterations.

Source: Own calculations based on HRS, SSA and HMD data. 


\section{References}

Baron, J. (2007). Thinking and Decision. New York, Melbourne, Madrid: Cambridge University Press.

Bloom, D. E., D. Canning, M. Moore, and Y. Song (2006). The Effect of Subjective Survival Probabilities on Retirement and Wealth in the United States. NBER Working Paper 12688.

Börsch-Supan, A. (1998). Comment on 'Subjective Survival Curves and Life Cycle Behavior'. In D. A. Wise (Ed.), Inquiries in the Economics of Aging, pp. 306-309. Chicago: University of Chicago Press.

Chateauneuf, A., J. Eichberger, and S. Grant (2007). Choice under Uncertainty with the Best and Worst in Mind: Neo-Additive Capacities. Journal of Economic Theory. forthcoming.

Eichberger, J., S. Grant, and D. Kelsey (2006). Updating Choquet Expected Utility Preferences. mimeo.

Eichberger, J. and D. Kelsey (1999). E-Capacities and the Ellsberg Paradox. Theory and Decision 46, 107-140.

Ellsberg, D. (1961). Risk, Ambiguity and the Savage Axioms. Quarterly Journal of Economics 75, 643-669.

Epstein, L. G. (1999). A Definition of Uncertainty Aversion. Review of Economic Studies 66, 579-608.

Gan, L., M. Hurd, and D. McFadden (2003). Individual Subjective Survival Curves. NBER Working Paper No. 9480.

Ghirardato, P. and M. Marinacci (2002). Ambiguity Made Precise: A Comparative Foundation. Journal of Economic Theory 102, 251-289.

Gilboa, I. and D. Schmeidler (1993). Updating Ambiguous Beliefs. Journal of Economic Theory 59, 33-49.

Greene, W. (2008). Econometric Analysis. New Jersey: Prentice Hall.

Hammermesh, D. S. (1985). Expectations, Life Expectancy, and Economic Behavior. Quarterly Journal of Economics 100(2), 389-408.

Hill, D., M. Perry, and R. J. Willis (2004). Estimating Knightian Uncertainty from Survival Probability Questions on the HRS. Working Paper, University of Michigan.

Hurd, M. D. and M. Kathleen (1995). Evaluation of the Subjective Probabilities of Survival in the Health and Retirement Study. Journal of Human Resources 30(0), S268-S292.

Hurd, M. D., S. Rohwedder, and J. Winter (2005). Subjective Probabilities of Survival: An International Comparison. Working Paper. 
Kastenbaum, R. (2000). The Psychology of Death. New York: Springer Publishing Company.

Khwaja, A., F. Sloan, and S. Chung (2006). The Relationship Between Individual Expectations and Behaviors: Evidence on Mortality Expectations and Smoking Decisions. Working Paper, Duke University.

Lee, R. D. and L. Carter (1992). Modeling and Forecasting U.S. Mortality. Journal of the American Statistical Association 87 (419), 659-671.

Manski, C. F. (2004). Measuring Expectations. Econometrica 72(5), 1329-1376.

Pires, C. P. (2002). A Rule for Updating Ambiguous Beliefs. Theory and Decision 53, $137-152$.

Savage, L. J. (1954). The Foundations of Statistics. New York, London, Sydney: John Wiley and Sons, Inc.

Schmeidler, D. (1989). Subjective Probability and Expected Utility Without Additivity. Econometrica 57, 571-587.

Siniscalchi, M. (2001). Bayesian Updating for General Maxmin Expected Utility Preferences. mimeo.

Siniscalchi, M. (2006). Dynamic Choice under Ambiguity. mimeo.

Smith, V. K., D. H. Taylor, and F. A. Sloan (2001). Longevity Expectations and Death: Can People Predict Their Own Demise? American Economic Review 91(4), 1126-1134.

Smith, V. K., D. H. Taylor, F. A. Sloan, F. R. Johnson, and W. H. Desvouges (2001). Do Smokers Respond to Health Shocks? The Review of Economics and Statistics 83(4), 675-687.

Tonks, I. (1983). Bayesian Learning and the Optimal Investment Decision of the Firm. The Economic Journal 93, 87-98.

Viscusi, W. K. (1985). A Bayesian Perspective on Biases in Risk Perception. Economics Letters 17, 59-62.

Viscusi, W. K. (1990). Do Smokers Underestimate Risks? Journal of Political Economy 98(6), 1253-1269.

Viscusi, W. K. (1991). Age Variations in Risk Perceptions and Smoking Decisions. Review of Economics and Statistics 73(4), 577-588.

Wakker, P. (2001). Testing and Characterizing Properties of Nonadditive Measures Through Violations of the Sure-Thing Principle. Econometrica 69, 1039-1059.

Wakker, P. (2004). On the Composition of Risk Preference and Belief. Psychological Review 111, 236-241. 


\section{B Separate Appendix}

We here describe in detail the steps taken to derive the equations in proposition 2 from proposition 1 using the assumptions made in subsection 2.3. First, under the assumptions that (i) $h \subseteq I_{h}$, (ii) $\mu_{n(h)}(m)=\pi(m)$ and $\mu_{n(h)}(j)=\pi(j)$, (iii) $\tilde{\pi}(m \mid j)=\phi^{m-j} \pi(m \mid j)$ and (iv) $j=h$, we can rewrite $\tilde{\pi}_{I_{h}}(m \mid j)$ in proposition 1 as

$$
\begin{aligned}
& \tilde{\pi}_{I_{h}}(m \mid j)=\gamma_{n(h)}^{*} \cdot \tilde{\pi}(m \mid j)+\left(1-\gamma_{n(h)}^{*}\right) \cdot \frac{\mu_{n(h)}(m)}{\mu_{n(h)}(j)} \Leftrightarrow \\
& \tilde{\pi}_{j}(m \mid j)=\left(\gamma_{j}^{*} \cdot \phi^{m-j}+\left(1-\gamma_{j}^{*}\right)\right) \cdot \pi(m \mid j)
\end{aligned}
$$

Second, using the additional assumptions that (i) $n(h)=n(j)=\psi j$ and (ii) $\tilde{\pi}(j) \phi^{j} \pi(j)$ we can rewrite $\gamma_{j}^{\star}$ as

$$
\begin{aligned}
\gamma_{j}^{*} & =\frac{\tilde{\pi}(j) \cdot(\alpha+\beta)}{\tilde{\pi}(j) \cdot(\alpha+\beta)+n(h) \cdot \pi(j)} \\
& =\frac{\phi^{j} \pi(j) \cdot(\alpha+\beta)}{\pi(j)\left(\phi^{j} \cdot(\alpha+\beta)+\psi j\right)} \\
& =\frac{\phi^{j}}{\phi^{j}+\frac{\psi}{\alpha+\beta} j} \\
& =\frac{\phi^{j}}{\phi^{j}+\xi j}
\end{aligned}
$$

Using the above in (16) we finally get

$$
\begin{aligned}
\tilde{\pi}_{j}(m \mid j) & =\left(\gamma_{j}^{*} \cdot \phi^{m-j}+\left(1-\gamma_{j}^{*}\right)\right) \cdot \pi(m \mid j) \\
& =\left(\frac{\phi^{j}}{\phi^{j}+\xi j} \cdot \phi^{m-j}+\left(1-\frac{\phi^{j}}{\phi^{j}+\xi j}\right)\right) \cdot \pi(m \mid j) \\
& =\frac{\phi^{m}+\xi j}{\phi^{j}+\xi j} \cdot \pi(m \mid j) .
\end{aligned}
$$




\section{Discussion Paper Series}

Mannheim Research Institute for the Economics of Aging Universität Mannheim

To order copies, please direct your request to the author of the title in question.

\begin{tabular}{|c|c|c|c|}
\hline Nr. & Autoren & Titel & Jahr \\
\hline $142-07$ & $\begin{array}{l}\text { Karsten Hank, } \\
\text { Stephanie Stuck }\end{array}$ & $\begin{array}{l}\text { Volunteer Work, Informal Help, and Care among } \\
\text { the 50+ in Europe: Further Evidence for 'Linked' } \\
\text { Productive Activities at Older Ages }\end{array}$ & 07 \\
\hline $143-07$ & Jürgen Maurer & $\begin{array}{l}\text { Assessing Horizontal Equity in Medication } \\
\text { Treatment Among Elderly Mexicans: Which } \\
\text { Socioeconomic Determinants Matter Most? }\end{array}$ & 07 \\
\hline $144-07$ & Jürgen Maurer & $\begin{array}{l}\text { Socioeconomic and Health Determinants of } \\
\text { Health Care Utilization Among Elderly } \\
\text { Europeans: A Semiparametric Assessment of } \\
\text { Equity, Intensity and Responsiveness for Ten } \\
\text { European Countries }\end{array}$ & 07 \\
\hline $145-07$ & Jürgen Maurer & $\begin{array}{l}\text { Modelling socioeconomic and health } \\
\text { determinants of health care use: } A \\
\text { semiparametric approach }\end{array}$ & 07 \\
\hline $146-07$ & $\begin{array}{l}\text { Jérome Adda, James } \\
\text { Banks, Hans-Martin } \\
\text { von Gaudecker }\end{array}$ & $\begin{array}{l}\text { The Impact of Income Shocks on Health: } \\
\text { Evidence from Cohort Data }\end{array}$ & 07 \\
\hline $147-07$ & $\begin{array}{l}\text { Klaus Jäger, } \\
\text { Wolfgang Kuhle }\end{array}$ & $\begin{array}{l}\text { The Optimum Growth Rate for Population } \\
\text { Reconsidered }\end{array}$ & 07 \\
\hline $148-07$ & $\begin{array}{l}\text { Axel Börsch-Supan, } \\
\text { Ismail Düzgün, } \\
\text { Matthias Weiss }\end{array}$ & & 07 \\
\hline $149-07$ & Matthias Weiss & & 07 \\
\hline $150-07$ & Matthias Weiss & & 07 \\
\hline $151-07$ & $\begin{array}{l}\text { Alexander Ludwig, } \\
\text { Thomas Schelkle, } \\
\text { Edgar Vogel }\end{array}$ & $\begin{array}{l}\text { Demographic Change, Human Capital and } \\
\text { Endogenous Growth }\end{array}$ & 07 \\
\hline $152-07$ & $\begin{array}{l}\text { Karsten Hank } \\
\text { Stephanie Stuck }\end{array}$ & $\begin{array}{l}\text { Gesellschaftliche Determinanten produktiven } \\
\text { Alterns in Europa }\end{array}$ & 07 \\
\hline $153-07$ & $\begin{array}{l}\text { Axel Börsch-Supan } \\
\text { Christina B. Wilke }\end{array}$ & $\begin{array}{l}\text { Szenarien zur mittel- und langfristigen } \\
\text { Entwicklung der Anzahl der Erwerbspersonen } \\
\text { und der Erwerbstätigen in Deutschland }\end{array}$ & 07 \\
\hline $154-07$ & $\begin{array}{l}\text { Alexander Ludwig, } \\
\text { Alexander Zimper }\end{array}$ & $\begin{array}{l}\text { A Parsimonious Model of Subjective Life } \\
\text { Expectancy }\end{array}$ & 07 \\
\hline
\end{tabular}

